\title{
Étude par la méthode d'homogénéisation des effets combinés de la rugosité de surface et de la rhéologie du lubrifiant sur le comportement d'un contact hydrodynamique
}

\author{
Ahcene Mouassa $^{1}$, Mustapha LahmaR ${ }^{1}$ et Benyebka Bou-Said ${ }^{2, a}$ \\ 1 Laboratoire de Mécanique et Structures (LMS), Université de Guelma, BP 401, Ouelma 2400, Algérie \\ 2 Université de Lyon, CNRS INSA-Lyon, LaMCoS, UMR 5259, 69621 Villeurbanne, France
}

Reçu le 19 janvier 2010, accepté le 23 mai 2010

Résumé - Dans cet article, les effets combinés de la rugosité de surface et de la rhéologie du lubrifiant additivé sur les performances hydrodynamiques d'un patin plan incliné sont étudiés au moyen de la méthode d'homogénéisation. La surface du patin contiguë au film est supposée fixe et rugueuse tandis que la surface inférieure mobile est parfaitement lisse. Le modèle de fluide polaire ou à couple de contrainte de V.K. Stokes est adopté pour décrire le comportement rhéologique du lubrifiant s'écoulant entre les deux surfaces. Les études de simulation sont effectuées en considérant trois formes de rugosités (transversales, longitudinales et anisotropes) et différentes valeurs du paramètre de couple de contrainte. La comparaison des solutions obtenues par les méthodes déterministe et d'homogénéisation a permis de conclure que la méthode d'homogénéisation est efficace pour les trois formes de rugosité envisagées. Les résultats de l'étude paramétrique effectuée montrent que les rugosités de surface et les couples de contraintes dus à la présence des additifs polymériques dans le lubrifiant ont des effets non négligeables sur les performances hydrodynamiques du contact, à savoir : le champ de pression, la capacité de charge, le nombre de frottement et la puissance dissipée.

\begin{abstract}
Mots clés : Lubrification hydrodynamique / rugosité de surface / patin incliné / fluide polaire / méthode d'homogénéisation / méthode déterministe

Abstract - A study of combined effects of surface roughness and lubricant rheology on the hydrodynamic contact behavior using the homogenization method. The combined effects of surface roughness and lubricant rheology on the performance characteristics of a hydrodynamic inclined slider bearing are investigated by means of the homogenized method. The pad surface is rough and stationary while the lower surface is assumed to be smooth and moving. The V.K. Stokes couple stress fluid model is adopted to describe the rheological behavior of the lubricant flowing between the two surfaces. The numerical simulations are performed by considering three roughness patterns (transverse, longitudinal and anisotropic), and various values of the couple stress parameter. It is found that the homogenization method is rigorous and efficient for the three roughness patterns considered. It is also found that the combined effects of the surface roughness as well as the couple stress due to the presence of polymer additives on the hydrodynamic performance characteristics such as pressure field, load carrying capacity, friction factor and power loss are significant.
\end{abstract}

Key words: Hydrodynamic lubrication / surface roughness / slider bearing / polar fluid / homogenization method / deterministic method

\section{Introduction}

La théorie de lubrification hydrodynamique classique fondée par Osborne Reynolds en 1886 est basée sur l'hypothèse de surfaces parfaitement lisses [1]. Cependant, il a été montré qu'une telle hypothèse est peu réaliste surtout

\footnotetext{
a Auteur pour correspondance :

benyebka.bou-said@insa-lyon.fr
}

pour l'étude des contacts sévèrement chargés fonctionnant avec de faibles épaisseurs de film.

Les pièces mécaniques manufacturées sont limitées par des surfaces qui ne sont jamais conformes à celles géométriquement idéales représentées sur les dessins de définition et de fabrication. En effet, elles présentent des défauts de nature géométriques et physico-chimiques résultant du procédé de fabrication utilisé et du matériau constituant ces pièces. 


\section{Nomenclature}

\begin{tabular}{|c|c|}
\hline $\bar{B}$ & largeur du patin $(\mathrm{m})$ \\
\hline$C_{\mathrm{f}}$ & nombre de frottement, \\
\hline$F$ & force de frottement $(\mathrm{N})$ \\
\hline$h_{0}$ & épaisseur du film du contact parfait (m) \\
\hline$h_{1}$ & amplitude des rugosités $(\mathrm{m})$ \\
\hline$h$ & épaisseur du film lubrifiant, $h=h_{0}+h_{1}(\mathrm{~m})$ \\
\hline$h_{\min }$ & épaisseur minimale du film à la sortie du contact, $h_{\min }=\min \left(h_{0}\right)(\mathrm{m})$ \\
\hline$h_{\max }$ & épaisseur maximale du film à l'entrée du contact, $h_{\max }=\max \left(h_{0}\right)(\mathrm{m})$ \\
\hline$h_{\varepsilon}$ & épaisseur déterministe du film lubrifiant (m) \\
\hline$L$ & longueur du patin $(\mathrm{m})$ \\
\hline$\underset{\sim}{\ell}$ & paramètre du couple des contraintes, $\ell=\sqrt{\eta / \mu}(\mathrm{m})_{\sim}$ \\
\hline$\tilde{\ell}$ & paramètre du couple des contraintes adimensionné, $\tilde{\ell}=\ell / h_{\min }$, \\
\hline$n_{\mathrm{p}}$ & nombre de périodes ou de rugosités, $n_{\mathrm{p}}=1 / \varepsilon$ \\
\hline$p$ & pression dans le film lubrifiant $(\mathrm{Pa})$ \\
\hline$p_{0}$ & pression, solution homogénéisée $(\mathrm{Pa})$ \\
\hline$p_{\varepsilon}$ & pression, solution déterministe $(\mathrm{Pa})$ \\
\hline$P$ & puissance dissipée, $W$ \\
\hline $\bar{Q}$ & débit de fuite moyen $\left(\mathrm{m}^{3} \cdot \mathrm{s}^{-1}\right)$ \\
\hline$r$ & rapport des épaisseurs du film à l'entrée et à la sortie du contact, $r=h_{\max } / h_{\min }>1$, \\
\hline$U$ & vitesse de déplacement de la glissière $\left(\mathrm{m} \cdot \mathrm{s}^{-1}\right)$ \\
\hline$u_{1}, u_{2}$ & composantes du champ de vitesse de l'écoulement suivant $x_{1}$ et $x_{2}\left(\mathrm{~m} . \mathrm{s}^{-1}\right)$ \\
\hline $\bar{u}_{1}, \bar{u}_{2}$ & vitesses moyennes de l'écoulement suivant $x_{1}$ et $x_{2}\left(\mathrm{~m} . \mathrm{s}^{-1}\right)$ \\
\hline$W$ & portance hydrodynamique $(\mathrm{N})$ \\
\hline$x$ & coordonnées d'espace globales, $\boldsymbol{x}=\left\langle x_{1} \quad x_{2}\right\rangle$, \\
\hline$y$ & coordonnées d'espace locales, $\boldsymbol{y}=\left\langle y_{1} \quad y_{2}\right\rangle$ \\
\hline$Y$ & cellule unitaire, $Y=(0,1) \times(0,1)$ \\
\hline$\alpha$ & amplitude des rugosités $(\mathrm{m})$ \\
\hline$\tilde{\alpha}$ & amplitude adimensionnée, $\tilde{\alpha}=\alpha / h_{\min }$ \\
\hline$\varepsilon$ & paramètre inversement proportionnel au nombre de périodes, $\varepsilon=1 / n_{\mathrm{p}}$, \\
\hline$\Phi$ & fonction de dissipation visqueuse $\left(\mathrm{W} \cdot \mathrm{m}^{-3}\right)$ \\
\hline$\eta$ & constante physique due à la présence des couples de contraintes (N.s) \\
\hline$\lambda$ & longueur d'onde des rugosités $(\mathrm{m})$ \\
\hline$\lambda_{x_{1}}, \lambda_{x_{2}}$ & longueurs d'onde des rugosités suivant les directions $x_{1}$ et $x_{2}(\mathrm{~m})$ \\
\hline$\Lambda$ & constante, $\Lambda=6 \mu U($ Pa.m) \\
\hline$\rho$ & masse volumique du lubrifiant $\left(\mathrm{kg} \cdot \mathrm{m}^{-3}\right)$ \\
\hline$\mu$ & viscosité dynamique du lubrifiant (Pa.s) \\
\hline$\vec{\nabla}_{x}(\cdot)$ & opérateur différentiel, $\vec{\nabla}_{x}(\cdot)=\left\langle\frac{\partial(\cdot)}{\partial x_{1}} \frac{\partial(\cdot)}{\partial x_{2}}\right\rangle$, \\
\hline$\vec{\nabla}_{y}(\cdot)$ & opérateur différentiel, $\vec{\nabla}_{y}(\cdot)=\left\langle\frac{\partial(\cdot)}{\partial y_{1}} \frac{\partial(\cdot)}{\partial y_{2}}\right\rangle$ \\
\hline$\vec{\nabla}(\cdot)$ & gradient de la fonction $(\cdot), \vec{\nabla}(\cdot)=\vec{\nabla}_{x}(\cdot)+\varepsilon^{-1} \vec{\nabla}_{y}(\cdot)$, \\
\hline$\vec{\nabla}$ & opérateur de divergence, \\
\hline$(\tilde{\sim})$ & quantité adimensionnée, \\
\hline$(\cdot)$ & quantité vectorielle, \\
\hline$(\cdot)$ & valeur moyenne, \\
\hline\langle\rangle & vecteur ligne, \langle\rangle$\equiv\{\}^{\mathrm{T}}$, \\
\hline Abréviations & \\
\hline EHD & élasto-hydro-dynamique, \\
\hline EDPs & équations aux dérivées partielles, \\
\hline RMS & moyenne quadratique des écarts du profil de rugosité par rapport à la ligne moyenne de rugosité \\
\hline
\end{tabular}


Selon la normalisation française (NF E 05-515), les défauts ou les irrégularités géométriques de surface se classent conventionnellement sous six numéros d'ordre, à savoir :

1. Défauts de forme et de position qui sont imputables à un manque de rectitude, à une ovalisation ou à un manque de cylindricité. Leur amplitude varie de 2,5 à plus de 100 microns et leur période est de plusieurs ordres de grandeur plus grande que leur amplitude;

2. défauts d'ondulation (défauts macroscopiques) possédant généralement un caractère périodique dû essentiellement à des vibrations de basses fréquences de la pièce et/ou de l'outil générateur. La période de ces défauts est de quelques ordres de grandeur plus grande que leur amplitude, soit de 0,1 à 50 microns ;

3. stries, sillons qui sont des défauts périodiques ou pseudo-périodiques ;

4. défauts localisés (échelle microscopique) : arrachement, marque d'outil, etc. : ce type de défaut a un caractère apériodique;

5. structure cristalline (échelle mésoscopique);

6. réseau cristallin (échelle nanoscopique).

Les défauts des $3^{\mathrm{e}}$ et $4^{\mathrm{e}}$ ordres, qui sont intéressants du point de vue tribologique, sont désignés par le terme général de rugosités. Il convient de préciser que le profil d'une surface mesuré à l'aide d'un profilographe est le résultat de la superposition des défauts de forme, d'ondulation et de rugosité.

Les amplitudes de rugosités peuvent varier de 0,01 microns ou moins pour les surfaces polies à 25 microns pour les surfaces usinées par des procédés conventionnels. Dans les systèmes mécaniques lubrifiés en régime hydrodynamique ou élasto-hydrodynamique (EHD) tels que les paliers, les butées, les engrenages et les roulements, l'épaisseur du film lubrifiant $h$ est censée d'une part être plus grande que la hauteur des aspérités et d'autre part elle doit être très faible devant la longueur d'onde de la rugosité (rugosité de Reynolds); c'est-à-dire $h \ll \lambda$. Dans ces conditions, l'équation de Reynolds peut être appliquée pour décrire le comportement hydrodynamique des contacts rugueux.

Plusieurs théories ont été proposées dans la littérature pour l'étude des effets des rugosités de surface sur les performances des contacts hydrodynamiques, à savoir les méthodes stochastiques et les méthodes déterministes. Par comparaison aux modèles stochastiques, les modèles déterministes permettent de prendre en compte de manière plus réaliste la texture et la microtopographie des surfaces dans les études de simulation.

\subsection{Méthode stochastique}

Le concept stochastique a été introduit pour la première fois par Tzeng et Saibel [2] en 1967 pour l'étude de l'influence d'une rugosité transversale sur les performances d'un patin incliné bidimensionnel.

En 1969, la théorie stochastique développée par Christensen [3] pour la lubrification hydrodynamique des surfaces rugueuses a été étendue au cas des paliers poreux par Prakash et Tiwari [4].

En 1971, Christensen et Tonder [5] développent l'équation moyennée pour analyser la répartition de la pression en présence de rugosités unidirectionnelles. Dans cette équation, ils introduisent le facteur de correction provenant de la théorie stochastique pour tenir compte des effets de la rugosité dans les patins.

En 1973, Elrod [6] a utilisé une approche stochastique pour mettre en évidence les effets des paramètres statistiques sur les caractéristiques d'un écoulement newtonien en film mince en prenant en compte les défauts de rugosité.

En 1978, Patir et Cheng [7] proposent une équation de Reynolds moyennée dans laquelle plusieurs facteurs de correction de débit sont introduits pour tenir compte des effets de la rugosité dans les 3 dimensions.

En 1997, Boedo et Booker [8] utilisent la méthode des éléments finis pour l'analyse des performances d'un palier avec une rugosité isotrope et ses effets sur la déformation élastique du palier. Dans cette étude, le fluide est supposé newtonien. En 2001, Wang et Keith [9] ont utilisé l'algorithme de cavitation universel développé par Elrod [10] en 1981 puis par Vijayaraghavan et Keith [11] pour analyser les effets de la rugosité ainsi que les effets non newtoniens sur le comportement de paliers dynamiquement chargés. Trois types de rugosité ont été étudiés : longitudinale, axiale et isotrope.

Récemment, Naduvinamani et al. [12] ont étudié l'effet de l'obliquité (skewness) de la rugosité sur les performances hydrodynamiques d'un patin échelon de Rayleigh poreux utilisant un fluide à couple de contrainte comme lubrifiant. Ils ont montré que pour une inclinaison négative de la rugosité, la capacité de charge du contact augmente tandis que le coefficient de frottement diminue. Cependant, la tendance inverse est observée pour une inclinaison positive.

\subsection{Méthode déterministe}

Ce type d'analyse est basé sur une résolution numérique directe de l'équation de Reynolds et une description de profils de surfaces simplifiées, en prenant par exemple un profil modélisé par une fonction périodique ou des profils réels (Fig. 1).

Il n'est plus nécessaire de connaître les paramètres statistiques relatifs au type de rugosité. Au lieu de considérer des facteurs de flux dans l'équation de Reynolds, on l'utilise sous la forme classique. C'est dans l'équation de l'épaisseur du film où sont prises en compte les rugosités.

En 1992, Kweh et al. [13] se sont intéressés au cas d'un contact lourdement chargé en introduisant une rugosité transverse sinusoïdale tridimensionnelle. Greenwood et al. [14] traitent le problème inverse; c'est-à-dire la rugosité de la surface est recherchée à partir des variations sinusoïdales de la pression.

La reproduction de la rugosité de la surface revient à regarder les variations de la pression et ne prendre en compte que les petites variations : les composantes 


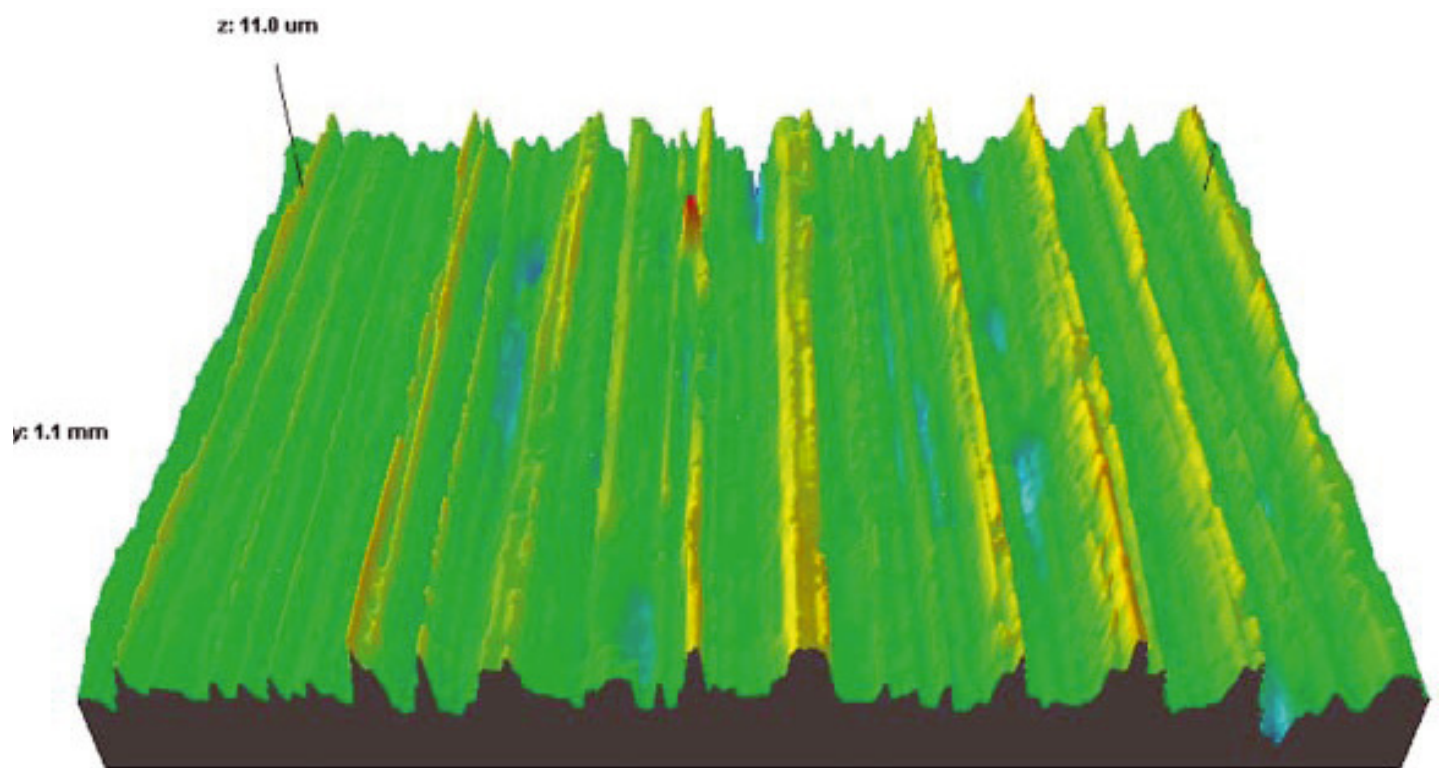

Fig. 1. Topographie réelle d'une surface d'acier abrasée unidirectionnellement.

de basses fréquences (l'ondulation) d'une rugosité de surface sont très aplaties alors que les composantes de hautes fréquences (rugosité) sont encore présentes après déformation.

En 1997, Zhu et al. [15] ont utilisé un relevé tridimensionnel de rugosité de surface pour étudier un contact ponctuel. Ils montrent que le pic de pression est une variable locale qui dépend largement des aspérités et de leurs pentes (obliquités).

L'approche déterministe permet de prendre en compte la variance la plus réaliste des rugosités. Les travaux effectués avec ce type d'approche sont basés sur l'application de l'équation de Reynolds et montrent qu'une description précise de la rugosité est une donnée importante. Elle permet d'obtenir des informations locales telles que les variations rapides locales de la pression.

Cependant, l'approche déterministe nécessite un espace mémoire important et de grands efforts de calcul car il faut raffiner au maximum le maillage pour pouvoir décrire les plus petites ondulations de la rugosité.

Récemment, Dobrica et al. [16] ont étudié l'influence de la pression spécifique sur les performances d'un palier à arc partiel rugueux de faibles dimensions dont la surface projetée est de $3 \mathrm{~cm}^{2}$ fonctionnant en régime de lubrification mixte. Pour prendre en considération les effets des différentes formes de rugosités (anisotropes, transversales et longitudinales), ces auteurs ont utilisé deux approches, à savoir : l'approche stochastique proposée par Patir et Cheng [7] basée sur la résolution d'une équation de Reynolds moyennée par la méthode des volumes finis et l'approche déterministe basée sur la résolution de l'équation de Reynolds classique au moyen de la même méthode, i.e. les volumes finis. Dans cette excellente étude, l'arbre tournant à une faible vitesse de rotation est supposé rigide et parfaitement lisse tandis que la surface du patin contiguë au film présente des rugosités gaussiennes isotropes ou orientées générées numériquement dont la rugosité $\mathrm{RMS}$ ou $R_{q}=$ 0,4 micron. Ils ont montré que :

- l'orientation de la rugosité de surface affecte les performances du palier tels que l'épaisseur minimale du film, l'angle de calage et les couples de frottement calculés sur l'arbre et le patin;

- par comparaison au cas lisse, l'effet de la rugosité transversale est le plus important conduisant à une augmentation importante de l'épaisseur minimale du film et du couple de frottement sur l'arbre:

- la rugosité longitudinale n'affecte que le couple de frottement.

À partir d'une comparaison des résultats obtenus par les deux approches déterministe et stochastique pour les trois formes de rugosités considérées, ils ont aussi montré que l'approche stochastique donne une bonne prédiction de l'épaisseur minimale du film et une mauvaise estimation (sous-estimation) des couples de frottement.

Plus récemment, les mêmes auteurs [17] ont développé une nouvelle approche élasto-hydrodynamique (EHD) pour l'étude du comportement des paliers à arc partiel rugueux fonctionnant en régime de lubrification mixte. Dans cette approche, l'arbre est supposé rigide et lisse tournant à de faibles vitesses tandis que le patin est rugueux et compliant dont le comportement est élastique parfaitement plastique (EPP).

\subsection{Méthode d'homogénéisation}

La méthode d'homogénéisation ou de moyennisation est une théorie mathématique qui inclut l'étude des équations aux dérivées partielles à coefficients à oscillation rapide [18]. Depuis quelques décennies, cette méthode a été utilisée avec succès pour le traitement des problèmes 
ayant trait, par exemple, à l'élasticité des milieux anisotropes tels que les matériaux composites [19,20]. En revanche, cette méthode n'est pas fréquemment utilisée dans le domaine de la tribologie. Récemment, plusieurs auteurs se sont intéressés à l'application du concept d'homogénéisation dans les études de simulation des problèmes de lubrification par fluides compressibles et incompressibles dont le comportement peut être newtonien ou non newtonien [21-29].

L'approche d'homogénéisation consiste à réécrire le problème posé en deux autres :

- Un problème local gouverné par 3 équations aux dérivées partielles du second ordre dont les inconnues sont les fonctions périodiques locales;

- Un problème homogénéisé régi par une équation aux dérivées partielles dont l'inconnue est la pression homogénéisée supposée nulle sur la frontière du domaine global.

La difficulté majeure de cette technique réside dans le découplage des deux problèmes à partir du problème global. Les coefficients du problème homogénéisé dépendent de la solution locale et ne peuvent être calculés qu'après traitement du problème local.

Par comparaison à la méthode déterministe décrite précédemment, la technique d'homogénéisation ne nécessite pas un maillage très fin pour prendre en compte l'effet de la rugosité car cette dernière est prise en compte pendant la résolution des problèmes locaux. Cependant, cette technique ne permet pas d'utiliser comme données les topographies de surfaces réelles mesurées suivant les 3 directions de l'espace.

À partir de la recherche bibliographique effectuée sur les différentes méthodes d'analyse, il ressort que la méthode d'homogénéisation s'avère être une alternative intéressante à la méthode déterministe spécifiquement dans le cas de rugosités périodiques car elle ne nécessite qu'une allocation mémoire réduite. De plus, elle présente des résultats plus réalistes comparés à ceux issus des approches stochastiques.

Dans le présent travail, nous appliquons la méthode d'homogénéisation pour l'analyse théorique des effets combinés des différentes formes de rugosités de surface (longitudinales, transversales et anisotropes) ainsi que la rhéologie du lubrifiant sur les performances hydrodynamiques d'un contact rugueux (patin incliné de Mitchell), à savoir : la capacité de charge, le nombre de frottement, la puissance dissipée et le débit de fuite. La surface du patin contiguë au film est supposée fixe et rugueuse tandis que la surface inférieure mobile (la glissière) est parfaitement lisse. Le patin est lubrifié à l'aide d'une huile minérale additivée dont le comportement rhéologique est non newtonien. Le modèle de fluide polaire ou à couple de contrainte de V.K. Stokes [30] est adopté pour décrire le comportement du lubrifiant s'écoulant entre les deux surfaces du contact. Ce type de fluide est caractérisé par deux constantes physiques qui sont la viscosité dynamique du lubrifiant $(\mu)$ et une deuxième caractéristique responsable du couple de contrainte dans le fluide $(\eta)$. Les effets de la rhéologie ou des couples de contrainte sur le comportement du patin seront étudiés en définissant le paramètre de couple de contrainte $(\ell=\sqrt{\eta / \mu})$ qui représente physiquement la longueur de la chaîne moléculaire des additifs incorporés à l'huile de base tels que les polymères améliorant l'indice de viscosité. À cause de sa simplicité mathématique, le modèle de fluide à couple de contrainte a été largement utilisé dans les études de simulation en lubrification hydrodynamique, hydrostatique, élasto-hydrodynamique et thermo-élasto-hydrodynamique [31-42].

\section{2 Équations de base de la lubrification hydrodynamique par fluide polaire}

\section{1 Équations de mouvement d'un fluide polaire}

En se basant sur la théorie des milieux continus de V.K. Stokes [30], les équations générales de mouvement d'un fluide incompressible à couple de contraintes (fluide polaire) dans le cas où les forces et les couples de volume sont négligées s'écrivent :

$$
\begin{aligned}
& \rho \frac{D \vec{V}}{D t}=-\vec{\nabla} p+\mu \vec{\nabla}^{2} \vec{V}-\eta \vec{\nabla}^{4} \vec{V} \\
& \vec{\nabla} \cdot \vec{V}=0
\end{aligned}
$$

où $\mu$ et $\eta$ sont respectivement la viscosité dynamique et la constante physique du fluide due à la présence des couples de contraintes.

En utilisant les hypothèses de la lubrification hydrodynamique établies pour ce type d'écoulement, les équations de mouvement de Stokes et l'équation de continuité s'écrivent en coordonnées cartésiennes comme suit [31] :

$$
\begin{aligned}
& \frac{\partial p}{\partial x_{i}}=\mu \frac{\partial^{2} u_{i}}{\partial x_{3}^{2}}-\eta \frac{\partial^{4} u_{i}}{\partial x_{3}^{4}} ; \quad i=1,2 \\
& \frac{\partial p}{\partial x_{3}}=0 \\
& \frac{\partial u_{j}}{\partial x_{j}}=0 ; \quad j=1,2,3
\end{aligned}
$$

\subsection{Expressions des vitesses de l'écoulement et de l'équation de Reynolds modifiée}

L'intégration des équations (3) et l'utilisation des conditions limites liées au non glissement des particules de fluide et à l'annulation des couples de contraintes au niveau des parois permet d'exprimer les vitesses de l'écoulement d'un fluide polaire par [31] :

$$
\left\{\begin{aligned}
u_{1}= & U \frac{x_{3}}{h}+\frac{1}{2 \mu} \frac{\partial p}{\partial x_{1}}\left(x_{3}\left(x_{3}-h\right)+2 \ell^{2}\right. \\
& \left.\times\left(1-\frac{\cosh \left(\frac{2 x_{3}-h}{2 \ell}\right)}{\cosh \left(\frac{h}{2 \ell}\right)}\right)\right) \\
u_{2}= & \frac{1}{2 \mu} \frac{\partial p}{\partial x_{2}}\left(x_{3}\left(x_{3}-h\right)+2 \ell^{2}\left(1-\frac{\cosh \left(\frac{2 x_{3}-h}{2 \ell}\right)}{\cosh \left(\frac{h}{2 \ell}\right)}\right)\right)
\end{aligned}\right.
$$




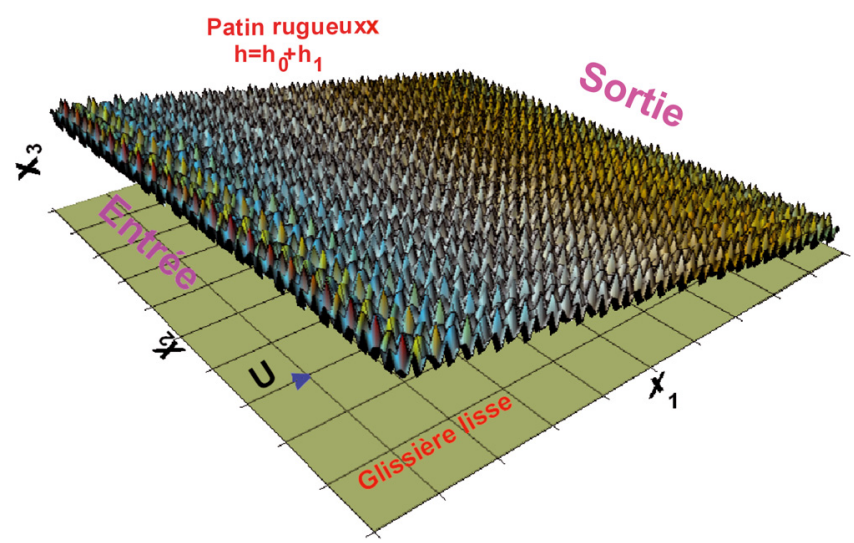

Fig. 2. Schéma du patin incliné rugueux.

où $\ell=\sqrt{\eta / \mu}$ est le paramètre du couple de contrainte qui représente physiquement la longueur de la chaîne moléculaire des additifs (dopes) incorporés.

Sachant que $u_{3}\left(x_{1}, x_{2}, 0\right)=u_{3}\left(x_{1}, x_{2}, h\right)=0$, la substitution des équations de la vitesse (5) dans l'équation de continuité (4) permet de dériver l'équation de Reynolds modifiée :

$$
\vec{\nabla} \cdot(G(h, \ell) \vec{\nabla} p)=\Lambda \frac{\partial h}{\partial x_{1}} \operatorname{sur} D=(0, L) \times(0, B)
$$

où

$$
G(h, \ell)=h^{3}-12 \ell^{2} h+24 \ell^{3} \tanh \left(\frac{h}{2 \ell}\right)
$$

et

$$
\Lambda=6 \mu U
$$

L'équation (6) décrit le comportement d'un fluide polaire non newtonien en régime d'écoulement stationnaire, isotherme et laminaire. Le champ de pression doit satisfaire à l'équation (6) et à la condition limite de Dirichlet suivante :

$$
p=0 \text { sur } \partial D
$$

\section{3 Équation géométrique du film}

La figure 2 représente schématiquement un patin incliné de Mitchell où seule la surface du patin fixe contiguë au film est rugueuse. Dans ces conditions, l'épaisseur du film s'exprime par :

$$
h\left(x_{1}, x_{2}\right)=h_{0}+h_{1}
$$

où :

$$
h_{0}=\left(h_{\min }-h_{\max }\right) \frac{x_{1}}{L}+h_{\max } \text { tel que } 0 \leq x_{1} \leq L
$$

et $h_{1}$ représente l'amplitude des rugosités mesurées par rapport au niveau moyen.

L'équation (10) donne l'épaisseur nominale du film définie comme la distance entre la glissière et le niveau moyen de la surface du patin.

\section{Performances hydrodynamiques du contact}

La détermination de la répartition de la pression dans le film lubrifiant $p$ pour une valeur du rapport $r=$ $h_{\max } / h_{\min }>1$ permet de déterminer les performances statiques du contact suivantes.

\subsection{Portance hydrodynamique}

La portance de l'écoulement est obtenue en intégrant le champ de pression hydrodynamique $p$ sur la surface du patin :

$$
W=\int_{0}^{L} \int_{0}^{B} p\left(x_{1}, x_{2}\right) \mathrm{d} x_{2} \mathrm{~d} x_{1}
$$

\subsection{Force de frottement}

L'intégration des contraintes de cisaillement à la surface de la glissière permet de calculer la force de frottement :

$$
\begin{aligned}
F & =\int_{0}^{L} \int_{0}^{B}\left(\mu\left[\frac{\partial u_{1}}{\partial x_{3}}\right]_{x_{3}=0}-\eta\left[\frac{\partial^{3} u_{1}}{\partial x_{3}^{3}}\right]_{x_{3}=0}\right) \mathrm{d} x_{2} \mathrm{~d} x_{1} \\
& =-\int_{0}^{L} \int_{0}^{B}\left(\mu \frac{U}{h}+\frac{h}{2} \frac{\partial p}{\partial x_{1}}\right) \mathrm{d} x_{2} \mathrm{~d} x_{1}
\end{aligned}
$$

\subsection{Nombre de frottement}

Le nombre de frottement est défini par :

$$
C_{\mathrm{f}}=\frac{|F|}{W}
$$

\subsection{Vitesses moyennes de l'écoulement et débit de fuite}

Les vitesses moyennes de l'écoulement et le débit de fuite moyen du patin sont calculés par :

$$
\begin{aligned}
& \left\{\begin{array}{l}
\bar{u}_{1}\left(x_{1}, x_{2}\right)=\frac{1}{h} \int_{0}^{h} u_{1}\left(x_{1}, x_{2}, x_{3}\right) \mathrm{d} x_{3}=\frac{U}{2}-\frac{G(h, \ell)}{12 \mu h} \frac{\partial p}{\partial x_{1}} \\
\bar{u}_{2}\left(x_{1}, x_{2}\right)=\frac{1}{h} \int_{0}^{h} u_{2}\left(x_{1}, x_{2}, x_{3}\right) \mathrm{d} x_{3}=-\frac{G(h, \ell)}{12 \mu h} \frac{\partial p}{\partial x_{2}}
\end{array}\right. \\
& \bar{Q}=\left|\bar{Q}_{0}\right|+\left|\bar{Q}_{B}\right|
\end{aligned}
$$

où

$$
\bar{Q}_{0}=\int_{0}^{L}\left[h \bar{u}_{2}\right]_{x_{3}=0} \mathrm{~d} x_{1} \text { et } \bar{Q}_{B}=\int_{0}^{L}\left[h \bar{u}_{2}\right]_{x_{3}=B} \mathrm{~d} x_{1}
$$

$\bar{Q}_{0}$ et $\bar{Q}_{B}$ étant les débits de fuite moyens aux extrémités libres du patin. 
Tableau 1. Expressions des différentes rugosités en coordonnées globales (macroscopiques).

\begin{tabular}{lcc}
\hline Types de rugosités & Expressions de $h_{1}$ & $\operatorname{rms}=\sqrt{\frac{1}{L B} \int_{0}^{B} \int_{0}^{L} h_{1}^{2}\left(x_{1}, x_{2}\right) \mathrm{d} x_{1} \mathrm{~d} x_{2}}$ \\
\hline Rugosités transversales & $h_{1}=\alpha \sin \left(\frac{2 \pi n_{\mathrm{p}} x_{1}}{L}\right)=\alpha \sin \left(\frac{2 \pi x_{1}}{\lambda_{x_{1}}}\right)$ & $\mathrm{rms}=\alpha \sqrt{2} / 2$ \\
\hline où $\lambda_{x_{1}}=\frac{L}{n_{\mathrm{p}}}=\varepsilon L$ et $\varepsilon=1 / n_{\mathrm{p}}$ & $\mathrm{rms}=\alpha \sqrt{2} / 2$ \\
\hline Rugosités longitudinales & $h_{1}=\alpha \sin \left(\frac{2 \pi n_{\mathrm{p}} x_{2}}{B}\right)=\alpha \sin \left(\frac{2 \pi x_{2}}{\lambda_{x_{2}}}\right)$ & $\mathrm{rms}=\alpha \sqrt{2} / 2$ \\
\hline où $\lambda_{x_{2}}=\frac{B}{n_{\mathrm{p}}}=\varepsilon B$ & $h_{1}=\alpha \sin \left(2 \pi n_{\mathrm{p}}\left(\frac{x_{1}}{L}+\frac{x_{2}}{B}\right)\right)=\alpha \sin \left(2 \pi\left(\frac{x_{1}}{\lambda_{x_{1}}}+\frac{x_{2}}{\lambda_{x_{2}}}\right)\right)$ & \\
\hline
\end{tabular}

\subsection{Puissance dissipée}

La puissance dissipée peut être calculée à partir de l'intégration de la fonction de dissipation visqueuse $\Phi[31]$ :

$$
P=\int_{0}^{L} \int_{0}^{B} \int_{0}^{h} \Phi\left(x_{1}, x_{2}, x_{3}\right) \mathrm{d} x_{3} \mathrm{~d} x_{2} \mathrm{~d} x_{1}
$$

où

$$
\begin{aligned}
\Phi= & \mu\left[\left(\frac{\partial u_{1}}{\partial x_{3}}\right)^{2}+\left(\frac{\partial u_{2}}{\partial x_{3}}\right)^{2}\right. \\
& \left.-\ell^{2}\left(\frac{\partial u_{1}}{\partial x_{3}} \frac{\partial^{3} u_{1}}{\partial x_{3}^{3}}+\frac{\partial u_{2}}{\partial x_{3}} \frac{\partial^{3} u_{2}}{\partial x_{3}^{3}}\right)\right]
\end{aligned}
$$

Lorsque $\ell$ tend vers zéro (cas newtonien), l'équation (18) se réduit à :

$$
\Phi=\mu\left[\left(\frac{\partial u_{1}}{\partial x_{3}}\right)^{2}+\left(\frac{\partial u_{2}}{\partial x_{3}}\right)^{2}\right]
$$

La puissance dissipée totale $P$ comprend deux termes correspondant aux effets de Hagen-Poiseuille et de Couette qui s'expriment comme suit :

$$
\begin{aligned}
P_{\text {Hagen-Poiseuille }}= & \int_{0}^{L} \int_{0}^{B} \frac{G(h, \ell)}{12 \mu}\left[\left(\frac{\partial p}{\partial x_{1}}\right)^{2}\right. \\
& \left.+\left(\frac{\partial p}{\partial x_{2}}\right)^{2}\right] \mathrm{d} x_{2} \mathrm{~d} x_{1} \\
P_{\text {Couette }}= & \int_{0}^{L} \int_{0}^{B} \frac{\mu U^{2}}{h} \mathrm{~d} x_{2} \mathrm{~d} x_{1}
\end{aligned}
$$

\section{Formulation mathématique et méthodes de résolution du problème de lubrification hydrodynamique des contacts rugueux}

\subsection{Méthode déterministe}

Cette méthode est basée sur une description de profils de surfaces simplifiée, en prenant par exemple un profil modélisé par une fonction périodique ou des profils réels (textures ou microtopographies des surfaces).

Dans cette méthode, l'équation de Reynolds modifiée (6) prend la forme suivante :

$$
\vec{\nabla} \cdot\left(G\left(h_{\varepsilon}, \ell\right) \vec{\nabla} p_{\varepsilon}\right)=\Lambda \frac{\partial h_{\varepsilon}}{\partial x_{1}} \operatorname{sur} D=(0, L) \times(0, B)
$$

où

$$
h_{\varepsilon}\left(x_{1}, x_{2}\right)=h_{0}\left(x_{1}, x_{2}\right)+h_{1}\left(x_{1} / \varepsilon, x_{2} / \varepsilon\right), \quad \varepsilon>0
$$

La pression déterministe $p_{\varepsilon}$ doit satisfaire à l'équation (22) et à la condition de Dirichlet suivante :

$$
p_{\varepsilon}=0 \text { sur la frontière } \partial D
$$

Dans l'équation (23), $h_{1}$ est une fonction périodique qui peut prendre, selon la forme des rugosités considérée, les expressions mathématiques indiquées dans le tableau 1.

Les trois formes de rugosités étudiées sont illustrées sur la figure 3.

L'équation (22) dont l'inconnue est la pression déterministe $p_{\varepsilon}$ n'admet pas de solution analytique, il est donc nécessaire d'utiliser les méthodes de résolution numériques.

La discrétisation de l'équation (22) par la méthode des différences finies centrées conduit au système d'équations algébriques suivant :

$a_{i j} p_{\varepsilon_{i+1, j}}+b_{i j} p_{\varepsilon_{i-1, j}}+c_{i j} p_{\varepsilon_{i, j+1}}+d_{i j} p_{\varepsilon_{i, j-1}}-e_{i j} p_{\varepsilon_{i j}}=f_{i j}$

où

$$
\begin{aligned}
& a_{i j}=\frac{G_{i j}}{\Delta x_{1}^{2}}+\frac{1}{2} \frac{\left(\frac{\partial G}{\partial x_{1}}\right)_{i j}}{\Delta x_{1}} ; b_{i j}=\frac{G_{i j}}{\Delta x_{1}^{2}}-\frac{1}{2} \frac{\left(\frac{\partial G}{\partial x_{1}}\right)_{i j}}{\Delta x_{1}} ; \\
& c_{i j}=\frac{G_{i j}}{\Delta x_{2}^{2}}+\frac{1}{2} \frac{\left(\frac{\partial G}{\partial x_{2}}\right)_{i j}}{\Delta x_{2}} ; d_{i j}=\frac{G_{i j}}{\Delta x_{2}^{2}}-\frac{1}{2} \frac{\left(\frac{\partial G}{\partial x_{2}}\right)_{i j}}{\Delta x_{2}} ; \\
& e_{i j}=2 G_{i j}\left(\frac{1}{\Delta x_{1}^{2}}+\frac{1}{\Delta x_{2}^{2}}\right) ; f_{i j}=\Lambda\left(\frac{\partial h}{\partial x_{1}}\right)_{i j}
\end{aligned}
$$

où $\Delta x_{i}$ sont respectivement les pas du maillage suivant les directions $x_{i}$. 
(a)

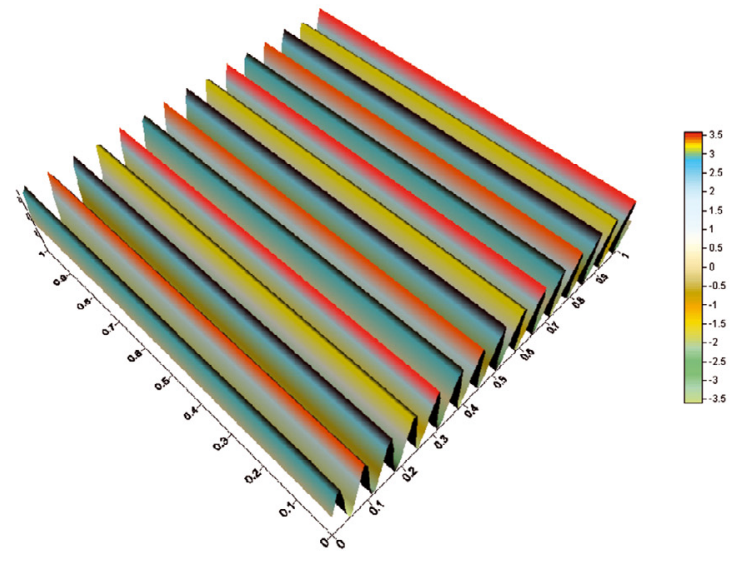

(b)

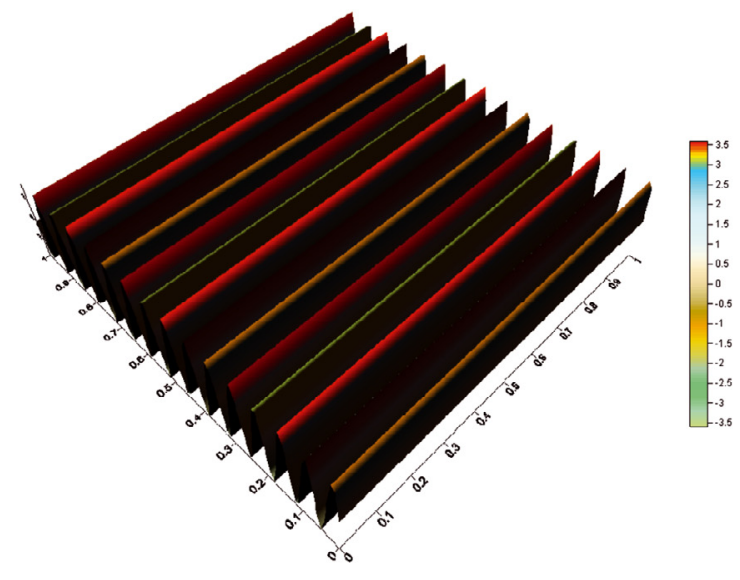

(c)

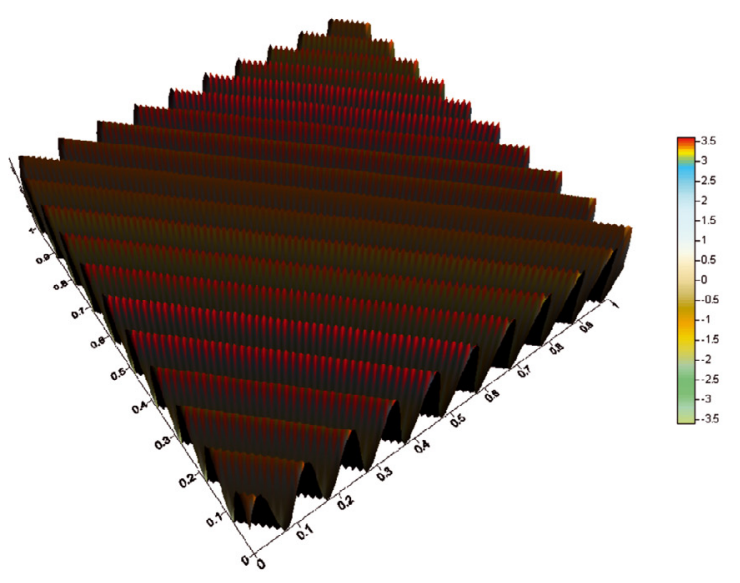

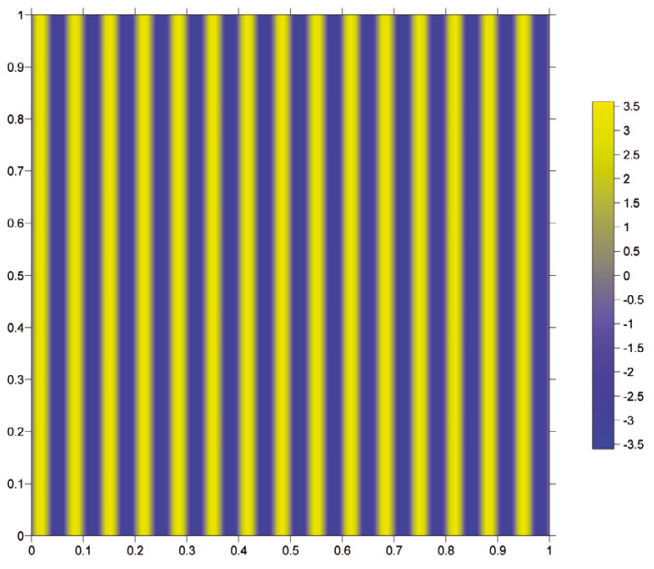
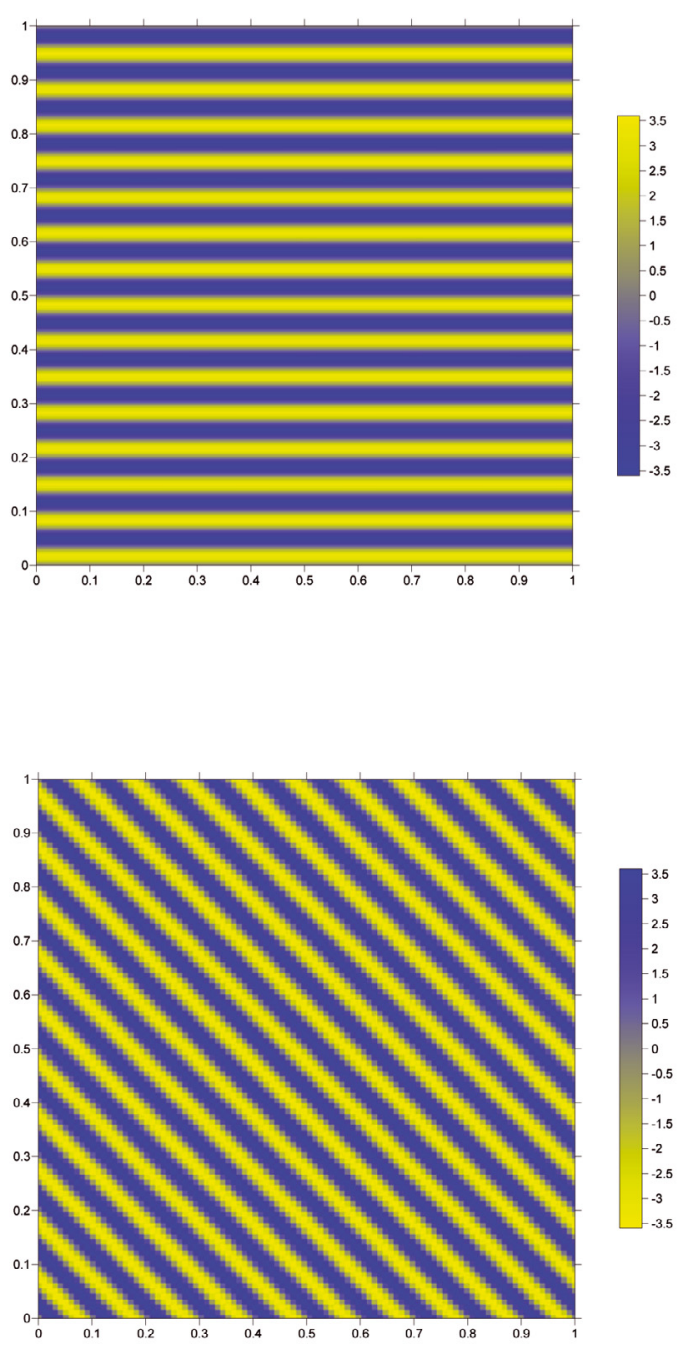

Fig. 3. Formes de rugosités étudiées. (a) Rugosités transversales. (b) Rugosités longitudinales. (c) Rugosités anisotropes. 
Tableau 2. Expressions des différentes rugosités en coordonnées locales (microscopiques).

\begin{tabular}{ccc}
\hline Types de rugosités & Expressions de $h_{1}$ & $\mathrm{rms}=\sqrt{\frac{1}{\Gamma Y} \int_{0}^{1} \int_{0}^{1} h_{1}^{2}\left(y_{1}, y_{2}\right) \mathrm{d} y_{1} \mathrm{~d} y_{2}}$ \\
\hline Rugosités transversales & $h_{1}=\alpha \sin \left(2 \pi y_{1}\right)$ & $\mathrm{rms}=\alpha \sqrt{2} / 2$ \\
Rugosités longitudinales & $h_{1}=\alpha \sin \left(2 \pi y_{2}\right)$ & $\mathrm{rms}=\alpha \sqrt{2} / 2$ \\
Rugosités anisotropes & $h_{1}=\alpha \sin \left(2 \pi\left(y_{1}+y_{2}\right)\right)$ & $\mathrm{rms}=\alpha \sqrt{2} / 2$ \\
\hline
\end{tabular}

Pour résoudre le système (25), il convient d'utiliser soit la méthode itérative de Gauss-Seidel avec facteur de sur-relaxation ou la méthode du gradient conjugué $[32,33]$.

\subsection{Méthode d'homogénéisation}

Le principe de cette méthode consiste à réécrire le problème de la lubrification des contacts rugueux en deux autres, à savoir : un problème local et un problème homogénéisé gouvernés par quatre équations aux dérivées partielles du second ordre.

Pour homogénéiser l'équation de Reynolds modifiée (6), on introduit la notion de coordonnées locales $\left(y_{1}, y_{2}\right)=\left(x_{1} / \varepsilon, x_{2} / \varepsilon\right)$ tel que $\left(y_{1}, y_{2}\right) \in Y=(0,1) \times$ $(0,1)$. L'équation géométrique (9) s'écrit alors :

$$
h\left(x_{1}, x_{2}, y_{1}, y_{2}\right)=h_{0}\left(x_{1}, x_{2}\right)+h_{1}\left(y_{1}, y_{2}\right)
$$

Les expressions de $h_{1}$ en fonction des coordonnées locales sont reportées dans le tableau 2.

Le développement asymptotique de la pression permet d'écrire :

$$
\begin{aligned}
p\left(x_{1}, x_{2}, y_{1}, y_{2}\right)= & p_{0}\left(x_{1}, x_{2}\right)+\varepsilon p_{1}\left(x_{1}, x_{2}, y_{1}, y_{2}\right) \\
& +\varepsilon^{2} p_{2}\left(x_{1}, x_{2}, y_{1}, y_{2}\right)+\ldots
\end{aligned}
$$

où $p_{1}$ et $p_{2}$ sont des fonctions périodiques par rapport aux variables $\left(y_{1}, y_{2}\right)$.

Nous utilisons la règle de différenciation suivante :

$$
\vec{\nabla}(\circ)=\vec{\nabla}_{x}(\circ)+\varepsilon^{-1} \vec{\nabla}_{y}(\circ)
$$

En limitant le développement de la pression $p$ à l'ordre un et après identification par rapport à $\varepsilon^{j}(j=0,-1,1)$, nous trouvons les équations suivantes:

$$
\begin{aligned}
& \vec{\nabla}_{x} \cdot\left(G(h, \ell)\left(\vec{\nabla}_{x} p_{0}+\vec{\nabla}_{y} p_{1}\right)\right)=\Lambda \frac{\partial h}{\partial x_{1}} \\
& -\vec{\nabla}_{y} \cdot\left(G(h, \ell) \vec{\nabla}_{x} p_{1}\right) \\
& \vec{\nabla}_{y} \cdot\left(G(h, \ell) \vec{\nabla}_{y} p_{1}\right)=\Lambda \frac{\partial h}{\partial y_{1}}-\vec{\nabla}_{y} \cdot\left(G(h, \ell) \vec{\nabla}_{x} p_{0}\right) \\
& \vec{\nabla}_{x} \cdot\left(G(h, \ell) \vec{\nabla}_{x} p_{1}\right)=0
\end{aligned}
$$

Pour découpler les équations (29) à (31), nous posons $[24]$ :

$$
\begin{aligned}
p_{1}\left(x_{1}, x_{2}, y_{1}, y_{2}\right)= & v_{1}\left(x_{1}, x_{2}, y_{1}, y_{2}\right) \frac{\partial p_{0}}{\partial x_{1}} \\
& +v_{2}\left(x_{1}, x_{2}, y_{1}, y_{2}\right) \frac{\partial p_{0}}{\partial x_{2}} \\
& +v_{3}\left(x_{1}, x_{2}, y_{1}, y_{2}\right)
\end{aligned}
$$

où $v_{1}, v_{2}$ et $v_{3}$ sont des fonctions périodiques.

En remplaçant l'équation (32) dans l'équation (30) et après identification, nous trouvons les équations différentielles suivantes :

$$
\begin{aligned}
& \vec{\nabla}_{y} \cdot\left(G(h, \ell) \vec{\nabla}_{y} v_{1}\right)=-\frac{\partial G}{\partial y_{1}} \operatorname{sur} Y \\
& \vec{\nabla}_{y} \cdot\left(G(h, \ell) \vec{\nabla}_{y} v_{2}\right)=-\frac{\partial G}{\partial y_{2}} \operatorname{sur} Y \\
& \vec{\nabla}_{y} \cdot\left(G(h, \ell) \vec{\nabla}_{y} v_{3}\right)=\Lambda \frac{\partial h}{\partial y_{1}} \operatorname{sur} Y
\end{aligned}
$$

où $Y=(0,1) \times(0,1)$ est la cellule unitaire.

L'intégration de l'équation (29) sur la cellule $Y$ conduit à :

$$
\vec{\nabla}_{x} \cdot\left(\boldsymbol{A}(\boldsymbol{x}) \vec{\nabla} p_{0}\right)=\vec{\nabla}_{x} \cdot \boldsymbol{\theta}(\boldsymbol{x})
$$

avec :

$$
\boldsymbol{A}=\left[\begin{array}{ll}
a_{11}\left(x_{1}, x_{2}\right) & a_{12}\left(x_{1}, x_{2}\right) \\
a_{21}\left(x_{1}, x_{2}\right) & a_{22}\left(x_{1}, x_{2}\right)
\end{array}\right] \text { et } \boldsymbol{\theta}=\left\{\begin{array}{l}
\theta_{1}\left(x_{1}, x_{2}\right) \\
\theta_{2}\left(x_{1}, x_{2}\right)
\end{array}\right\}
$$

Les coefficients $a_{i j}$ et $\theta_{i}$ sont définis par

$$
\begin{aligned}
a_{11} & =\int_{Y} G\left(1+\frac{\partial v_{1}}{\partial y_{1}}\right) \mathrm{d} Y ; a_{12}=\int_{Y} G \frac{\partial v_{2}}{\partial y_{1}} \mathrm{~d} Y \\
a_{21} & =\int_{Y} G \frac{\partial v_{1}}{\partial y_{2}} \mathrm{~d} Y ; \quad a_{22}=\int_{Y} G\left(1+\frac{\partial v_{2}}{\partial y_{2}}\right) \mathrm{d} Y \\
\theta_{1} & =\int_{Y}\left(\Lambda h-G \frac{\partial v_{3}}{\partial y_{1}}\right) \mathrm{d} Y \quad ; \quad \theta_{2}=-\int_{Y} G \frac{\partial v_{3}}{\partial y_{2}} \mathrm{~d} Y
\end{aligned}
$$

avec $\mathrm{d} Y=\mathrm{d} y_{1} \mathrm{~d} y_{2}$

La pression homogénéisée $p_{0}$ doit satisfaire à l'équation (36) et à la condition de Dirichlet:

$$
p_{0}=0 \text { sur la frontière } \partial D
$$


Tableau 3. Caractéristiques géométriques et conditions de fonctionnement du patin.

\begin{tabular}{|c|c|c|c|}
\hline Paramètres & Symboles & Valeurs & Unités \\
\hline Longueur du patin & $L$ & 0,10 & $\mathrm{~m}$ \\
\hline Largeur du patin & $B$ & 0,10 & $\mathrm{~m}$ \\
\hline Épaisseur minimale du film lubrifiant à la sortie du contact & $h_{\min }$ & 4. $\times 10^{-6}$ & $\mathrm{~m}$ \\
\hline Épaisseur maximale du film lubrifiant à l'entrée du contact & $h_{\max }$ & 8. $\times 10^{-6}$ & $\mathrm{~m}$ \\
\hline Amplitude des rugosités & $\alpha$ & $\begin{array}{l}0,0 \text { (Cas lisse) } \\
1 . \times 10^{-6}\end{array}$ & $\mathrm{~m}$ \\
\hline Écart quadratique moyen des rugosités & RMS & $\sqrt{2} / 2 \times 10^{-6}$ & $\mathrm{~m}$ \\
\hline Vitesse de déplacement de la glissière & $U$ & 1. & $\mathrm{~m} \cdot \mathrm{s}^{-1}$ \\
\hline $\begin{array}{l}\text { Viscosité dynamique du fluide lubrifiant } \\
\text { (huile industrielle ISO VG 32) }\end{array}$ & $\mu$ & $30 . \times 10^{-3}$ & Pa.s \\
\hline $\begin{array}{l}\text { Constante physique du fluide responsable du couple de contrainte } \\
\text { du couple de contrainte }\end{array}$ & $\eta$ & $\begin{array}{c}0,0 \text { (Cas newtonien) } \\
0,48 \times 10^{-4} \\
4,32 \times 10^{-3}\end{array}$ & N.s \\
\hline
\end{tabular}

Tableau 4. Caractéristiques des maillages par différences finies.

\begin{tabular}{llc}
\hline Méthodes d'analyse & EDPs gouvernant le problème & Nombre de mailles \\
\hline Déterministe & Équation de Reynolds (Éq. (22)) & $600 \times 600$ \\
Homogénéisation & Équations locales (Éqs. (33), (34) et (35)) & $40 \times 40$ \\
& Équation globale (Éq. (36)) & $80 \times 80$ \\
\hline
\end{tabular}

L'algorithme de la méthode d'homogénéisation comprend les étapes suivantes :

Étape 1 : résolution par la méthode des différences finies du problème local gouverné par le système d'équations (33), (34) et (35) dont les inconnues sont les fonctions périodiques $v_{1}(\boldsymbol{x}, \boldsymbol{y}), v_{2}(\boldsymbol{x}, \boldsymbol{y})$ et $v_{3}(\boldsymbol{x}, \boldsymbol{y})$;

Étape 2 : calcul des coefficients $a_{i j}(\boldsymbol{x})$ et $\theta_{i}(\boldsymbol{x}) ; i, j=$ 1,2 ;

Étape 3 : résolution par la méthode des différences finies du problème global régi par l'équation homogénéisée (36) dont l'inconnue est la pression homogénéisée $p_{0}$.

Il est utile de noter que les étapes 1 et 2 de l'algorithme d'homogénéisation s'appliquent à chaque point $\boldsymbol{x}$ du domaine global $(D)$.

\section{Résultats numériques et discussion}

Dans ce paragraphe, nous allons comparer les solutions obtenues par les deux méthodes d'analyse déterministe et d'homogénéisation développées précédemment et étudier en utilisant la méthode d'homogénéisation les effets non newtoniens du lubrifiant et des différentes formes de rugosités de surfaces sur les performances hydrodynamiques du contact, à savoir : la pression, la capacité de charge, la puissance dissipée, le nombre de frottement et le débit de fuite. Les données de cette étude sont reportées dans le tableau 3.

\subsection{Comparaison entre les solutions déterministes et homogénéisées}

Deux programmes de calcul ont été élaborés pour simuler le comportement du patin utilisant un fluide polaire comme lubrifiant en se basant sur le concept des deux méthodes d'analyse tridimensionnelles développées. Les solutions déterministes et homogénéisées, $p_{\varepsilon}$ et $p_{0}$, sont comparées dans le cas newtonien pour différentes formes ou modèles de rugosités qui peuvent être transversales, longitudinales et anisotropes, ainsi que pour diverses valeurs de l'amplitude relative de rugosité $(\tilde{\alpha}=0,0,0,025,0,25)$ et du paramètre $\varepsilon$ qui correspond à l'inverse du nombre de périodes ou de rugosités dont les valeurs sont $n_{p}=5,10$ et 40 . Notons que la valeur nulle de $\alpha$ correspond au cas du patin parfaitement lisse.

Tous les calculs sont effectués pour une valeur du rapport $r$ égale à 2. Les caractéristiques de maillages utilisés pour la discrétisation spatiale par la méthode des différences finies centrées des différentes équations aux dérivées partielles $(22,33,34,35$ et 36$)$ sont indiquées dans le tableau 4.

Les figures 4 et 5 représentent les répartitions de pression hydrodynamique obtenues dans le cas du patin lisse et du patin rugueux. D'après ces figures, nous observons nettement que les solutions obtenues dans le cas lisse sont confondues ce qui permet de valider le programme de calcul basé sur l'homogénéisation de l'équation de Reynolds. Dans le cas rugueux, nous remarquons que la solution déterministe obtenue pour une amplitude de rugosité relativement faible converge vers la solution homogénéisée et ce pour les trois formes de rugosités considérées. Cependant, la convergence de la solution déterministe ne peut être obtenue pour les formes de rugosités transversales et anisotropes que pour un nombre important de rugosités ; c'est-à-dire lorsque $\varepsilon \rightarrow 0$. Il est à noter que les rugosités longitudinales n'ont pas d'effets sur la convergence de la solution déterministe vers la solution homogénéisée du fait que ces défauts sont orientés dans le même sens 
a) Solution homogénéisée, $p_{0}$

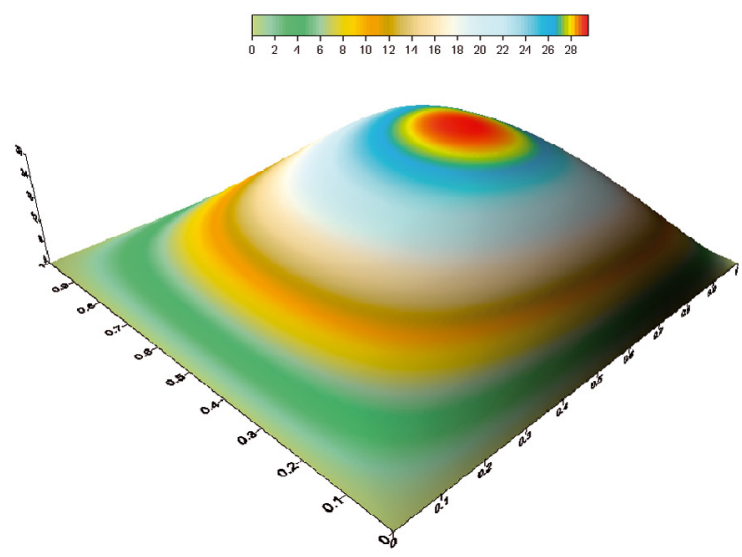

b) Solution déterministe, $p_{\varepsilon}$

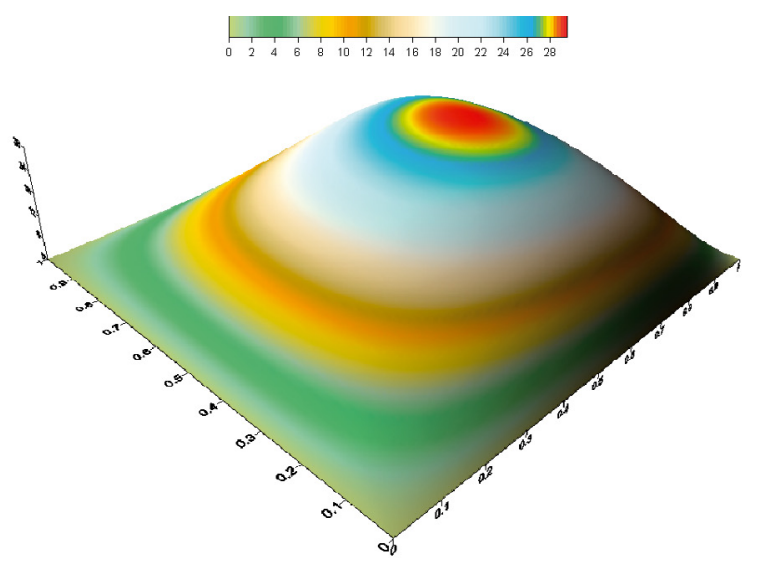

Représentations 3-D
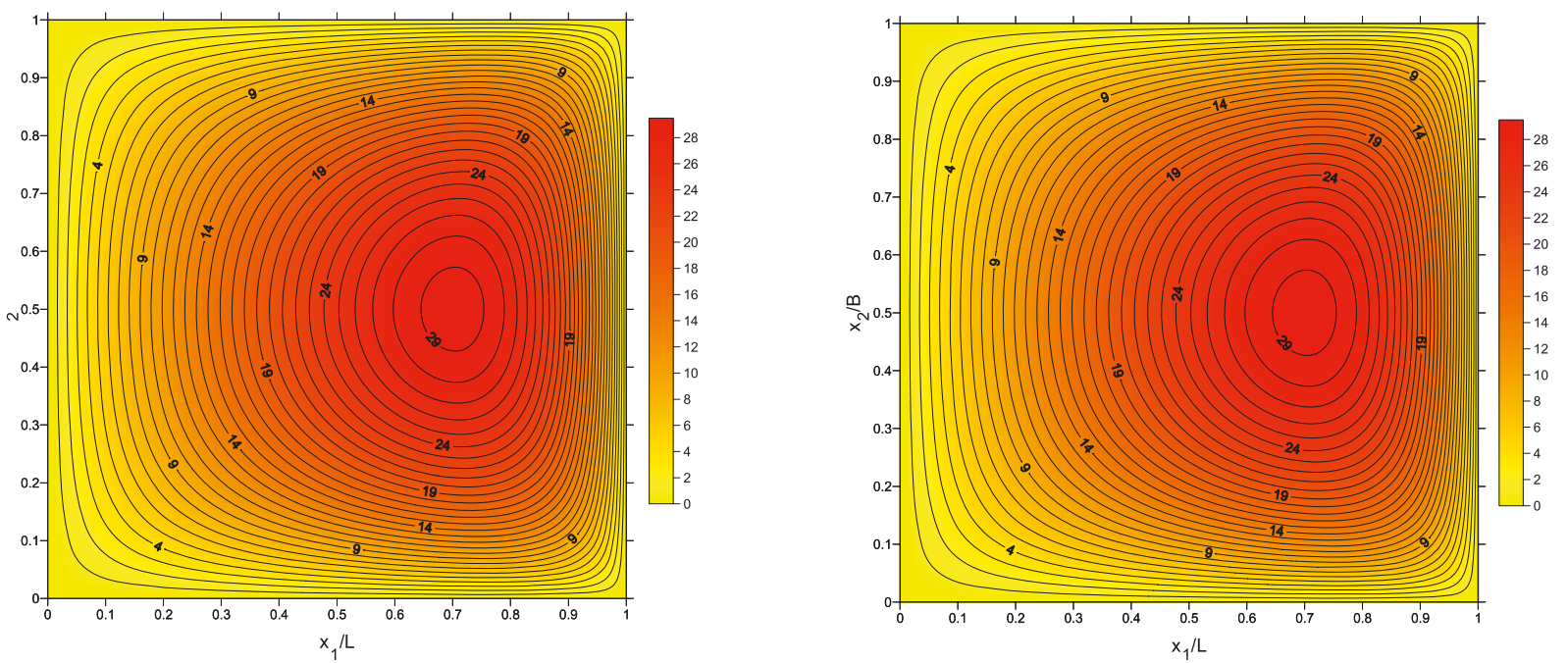

Lignes isobares

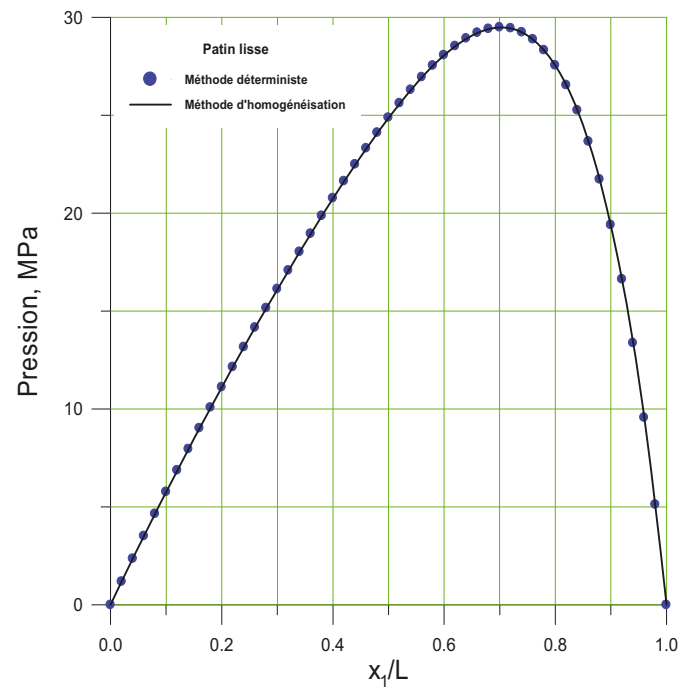

Répartition du champ de pression dans la section médiane du patin

Fig. 4. Répartitions des pressions homogénéisée et déterministe dans le cas du patin lisse $(\alpha=0,0, r=2, \ell=0)$. 
a) $\tilde{\alpha}=0.025, r=2$
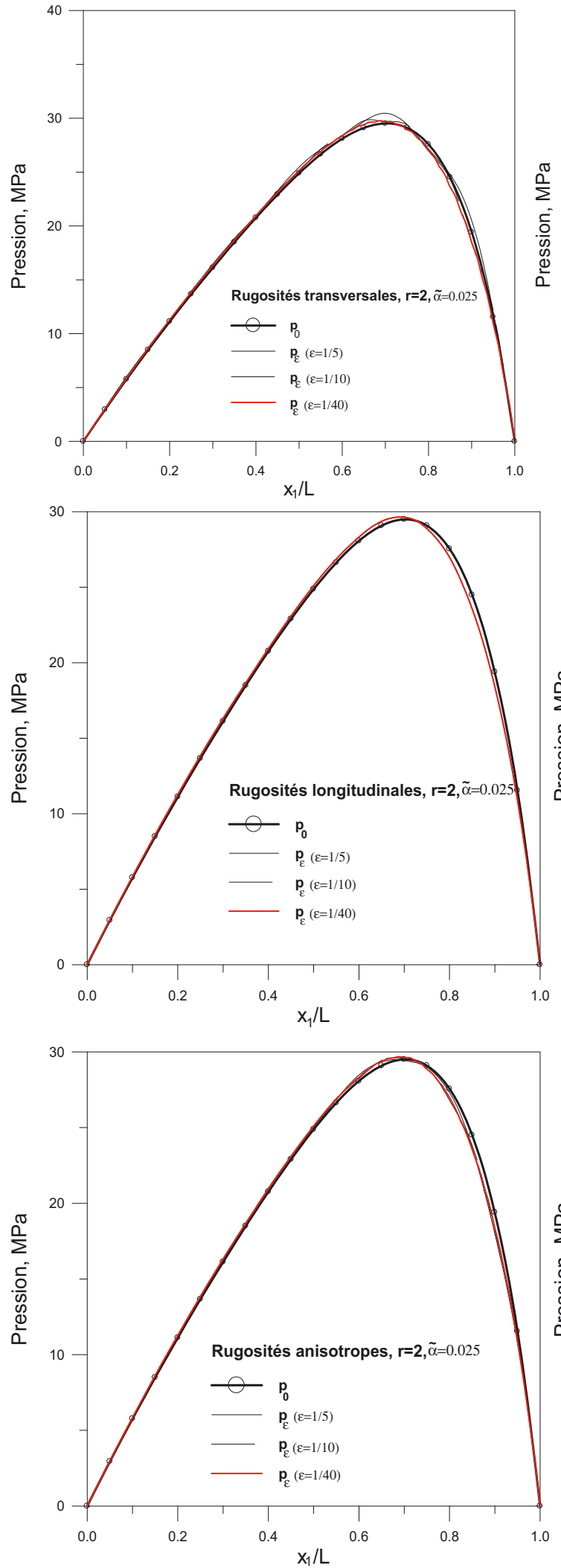

b) $\tilde{\alpha}=0.25, r=2$
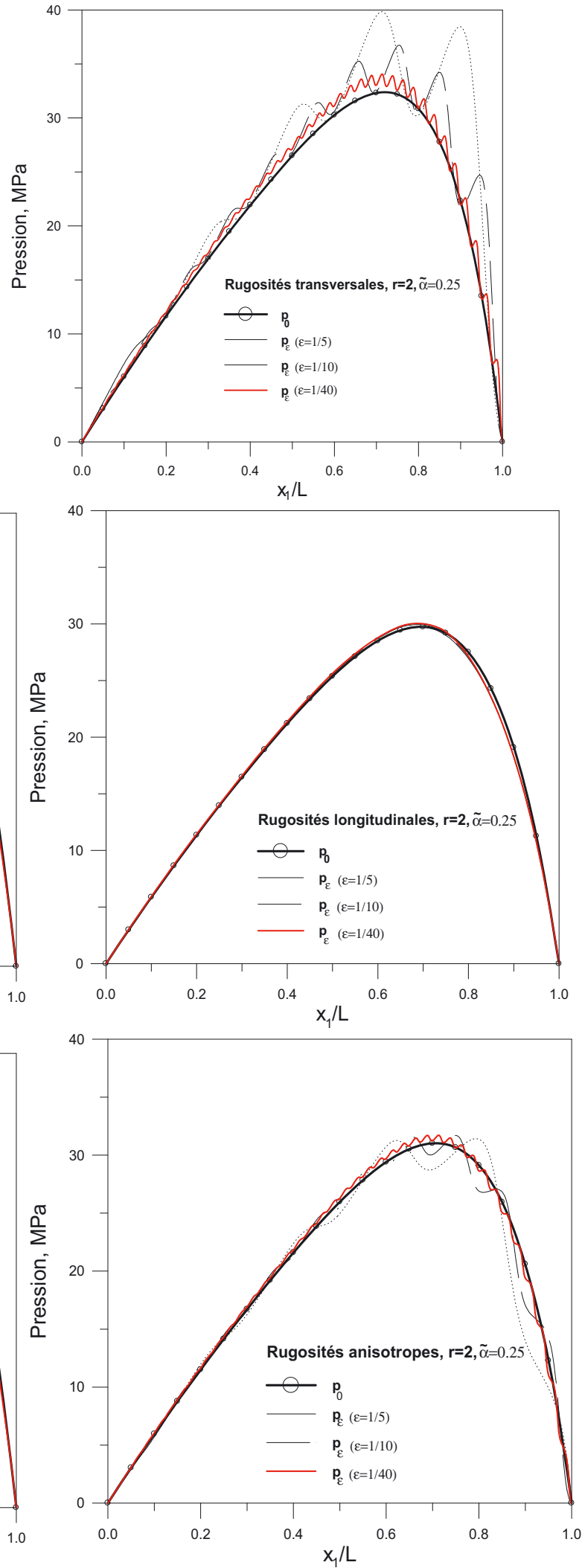

Fig. 5. Comparaison des solutions homogénéisées et déterministes pour les différentes formes de rugosités (patin rugueux). 
Tableau 5. Comparaison en temps CPU des méthodes déterministes et d’homogénéisation (Processeur Intel Pentium 1,66 GHz, RAM $503 \mathrm{MB})$.

\begin{tabular}{|c|c|c|c|c|}
\hline & & \multicolumn{3}{|c|}{ Modèles de rugosités } \\
\cline { 3 - 5 } Méthodes & Cas lisse & Transversales & Longitudinales & Anisotropes \\
d'analyse & $(\tilde{\alpha}=0)$ & $(\varepsilon=1 / 40, \tilde{\alpha}=1 / 4)$ & $(\varepsilon=1 / 40, \tilde{\alpha}=1 / 4)$ & $(\varepsilon=1 / 40, \tilde{\alpha}=1 / 4)$ \\
\hline Déterministe & $44,85 \mathrm{~s}$ & $56,53 \mathrm{~s}$ & $53,23 \mathrm{~s}$ & $56,0 \mathrm{~s}$ \\
Homogénéisation & $382,98 \mathrm{~s}$ & $487,52 \mathrm{~s}$ & $425,9 \mathrm{~s}$ & $476,35 \mathrm{~s}$ \\
\hline
\end{tabular}

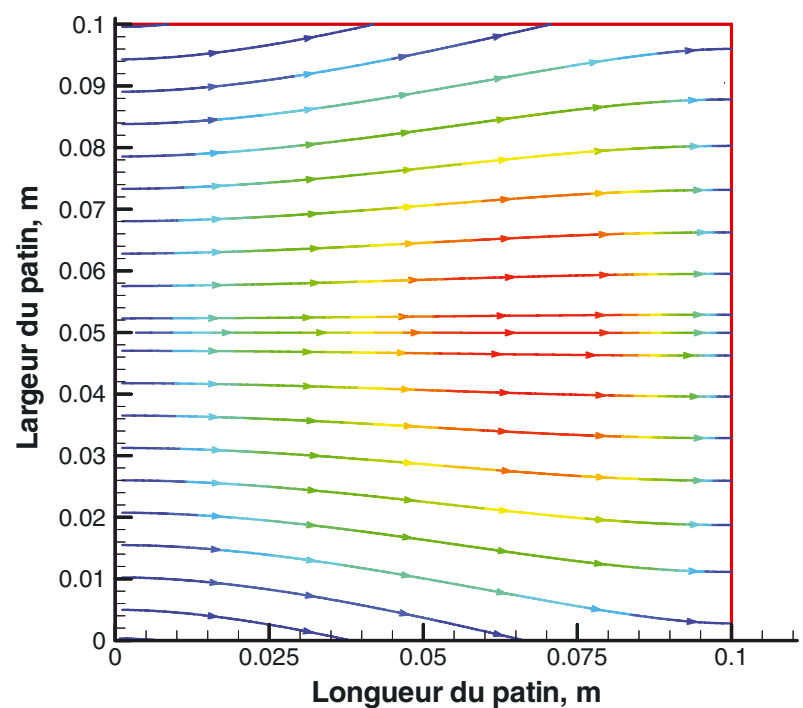

Fig. 6. Représentation des lignes de courant de l'écoulement calculées par la technique d'homogénéisation dans le cas des rugosités longitudinales $(\tilde{\alpha}=0,25, r=2)$.

que celui de l'écoulement du lubrifiant comme l'illustre la figure 6 .

La figure 7 illustre que lorsque le nombre de périodes ou de rugosités augmente l'écart relatif entre les portances hydrodynamiques déterministes et homogénéisées $\delta \tilde{W}=\int_{D}\left|p_{0}-p_{\varepsilon}\right| \mathrm{d} x_{1} \mathrm{~d} x_{2} / \int_{D} p_{0} \mathrm{~d} x_{1} \mathrm{~d} x_{2}$ diminue pour les trois formes de rugosités considérées. Cette diminution est plus rapide dans le cas des rugosités longitudinales.

Sur la base de ces résultats, nous pouvons conclure que pour des amplitudes de rugosités relativement faibles et un nombre important de rugosités, la technique d'homogénéisation, de mise en œuvre simple, est efficace du point de vue espace mémoire quelle que soit la forme de rugosité considérée. Néanmoins, l'application de cette technique n'est possible que si la distribution des rugosités sur la surface de contact est périodique. Ces constatations sont en bonne concordance avec celles de Kane et Bou-Saïd [26].

Dans le tableau 5, nous comparons les temps CPU exprimés en secondes des calculs effectués pour la détermination des solutions déterministes et homogénéisées sur un PC doté d'un processeur de fréquence 1,66 GHz. Nous remarquons que le temps de calcul nécessaire pour la convergence de la solution homogénéisée est plus important que celui de la solution déterministe aussi bien dans le cas lisse que rugueux. Cela s'explique par le fait que la résolution du problème homogénéisé nécessite le calcul de nombreuses intégrales et ce pour chaque point du domaine global. Une étude de sensibilité des maillages est alors nécessaire afin de déterminer les tailles optimales des différents maillages.

\subsection{Effets des rugosités de surface et des couples de contrainte sur les performances hydrodynamiques du patin}

La figure 8 représente les évolutions des performances hydrodynamiques du contact en fonction de l'amplitude relative des rugosités pour les différentes formes de rugosité représentées sur la figure 3. Les calculs ont été effectués par la méthode d'homogénéisation pour trois valeurs du paramètre de couple de contrainte adimensionné, à savoir $\tilde{\ell}=0$., 0,1 et 0,3 . Nous observons que pour les rugosités transversales et anisotropes, la pression maximale, la portance, la puissance dissipée et le nombre de frottement sont sensiblement influencés par l'amplitude des rugosités aussi bien pour le cas newtonien que non newtonien. Cependant, ces paramètres ne sont pas affectés de façon significative par l'amplitude dans le cas des rugosités longitudinales. Les résultats obtenus montrent aussi que l'amplitude des rugosités n'a pas d'effet appréciable sur le débit de fuite quelle que soit la forme de rugosité. Il est intéressant de souligner que pour une même valeur de l'amplitude, la pression maximale et la portance hydrodynamique calculées dans le cas des rugosités transversales sont nettement supérieures à celles obtenues avec les rugosités anisotropes et longitudinales. Le tableau 6 donne une quantification des effets des différentes rugosités sur les performances du patin. Les valeurs de ces performances correspondent à deux valeurs de l'amplitude relative de la rugosité $\tilde{\alpha}=0$. (cas lisse) et $\tilde{\alpha}=0,25$ obtenues dans le cas newtonien $\tilde{\ell}=0$. et non newtonien $\tilde{\ell}=0,3$.

La figure 9 montre les variations des performances hydrodynamiques en fonction du rapport $r$ pour trois valeurs du paramètre de couple de contrainte adimensionné $\tilde{\ell}=0$., 0,1 et 0,3 dans le cas d'un patin lisse et d'un patin rugueux. Notons que l'épaisseur minimale du film à la sortie du contact est constante et prise égale à 4 microns pour tous les calculs. La variation du rapport $r$ est donc obtenue en faisant varier l'épaisseur maximale du film à 

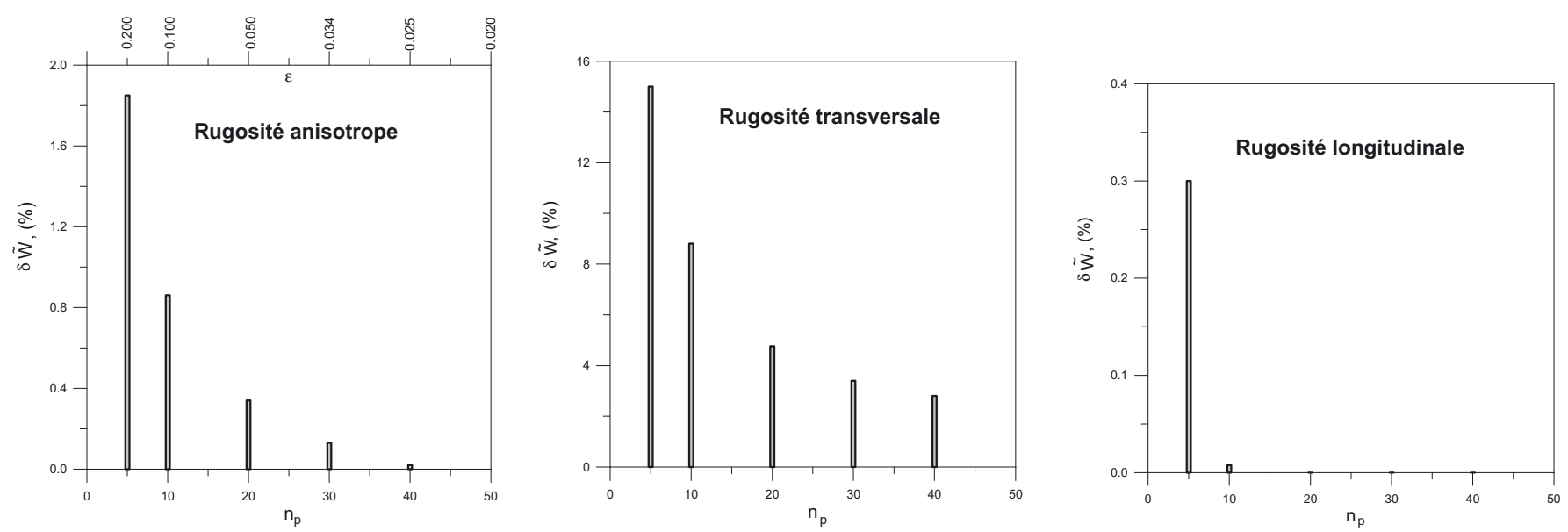

Fig. 7. Evolutions de l'écart relatif entre les capacités de charges déterministes et homogénéisées en fonction du nombre de périodes pour les trois formes de rugosités.

Tableau 6. Comparaisons des performances hydrodynamiques obtenues dans le cas lisse et rugueux pour deux valeurs du paramètre de couple de contrainte.

\begin{tabular}{|c|c|c|c|c|c|}
\hline \multirow{2}{*}{ Performances } & \multirow{2}{*}{ Type de rugosité } & \multicolumn{2}{|c|}{$\tilde{\ell}=0,0$} & \multicolumn{2}{|c|}{$\tilde{\ell}=0,3$} \\
\hline & & Valeur & $\begin{array}{c}\text { Écart } \\
\text { relatif } \\
(\%)\end{array}$ & Valeur & $\begin{array}{c}\text { Écart } \\
\text { relatif } \\
(\%)\end{array}$ \\
\hline \multirow{4}{*}{ Pression maximale $(\mathrm{MPa})$} & Cas lisse $(\tilde{\alpha}=0,0)$ & \multicolumn{2}{|c|}{29,5} & \multicolumn{2}{|c|}{45,1} \\
\hline & Transversale & 39,06 & $+32,4$ & 70,35 & +56 \\
\hline & Longitudinale & 30,00 & $+1,7$ & 45,00 & $-0,22$ \\
\hline & Anisotrope & 34,50 & $+16,9$ & 55,30 & $+22,62$ \\
\hline \multirow{4}{*}{ Portance $(\mathrm{kN})$} & Cas lisse $(\tilde{\alpha}=0,0)$ & \multicolumn{2}{|c|}{130} & \multicolumn{2}{|c|}{192} \\
\hline & Transversale & 163,0 & $+25,40$ & 267,4 & $+39,27$ \\
\hline & Longitudinale & 136,0 & $+4,61$ & 200,0 & $+4,20$ \\
\hline & Anisotrope & 148,4 & $+14,15$ & 229,1 & $+19,32$ \\
\hline \multirow{4}{*}{ Puissance dissipée (W) } & Cas lisse $(\tilde{\alpha}=0,0)$ & \multicolumn{2}{|c|}{54,6} & \multicolumn{2}{|c|}{55,8} \\
\hline & Transversale & 56,2 & $+2,93$ & 60,0 & $+7,53$ \\
\hline & Longitudinale & 54,8 & $+0,37$ & 56,2 & $+0,72$ \\
\hline & Anisotrope & 55,4 & $+1,46$ & 57,5 & $+3,05$ \\
\hline \multirow{4}{*}{ Nombre de frottement (-) } & Cas lisse $(\tilde{\alpha}=0,0)$ & \multicolumn{2}{|c|}{0,00042} & \multicolumn{2}{|c|}{0,00029} \\
\hline & Transversale & 0,00034 & $-19,05$ & 0,00021 & $-27,60$ \\
\hline & Longitudinale & 0,00040 & $-4,76$ & 0,00028 & $-3,45$ \\
\hline & Anisotrope & 0,00037 & $-11,90$ & 0,00025 & $-13,79$ \\
\hline \multirow{4}{*}{ Débit de fuite $\left(\mathrm{cm}^{3} . \mathrm{s}^{-1}\right)$} & Cas lisse $(\tilde{\alpha}=0,0)$ & \multicolumn{2}{|c|}{0,098} & \multicolumn{2}{|c|}{0,098} \\
\hline & Transversale & 0,113 & $+15,31$ & 0,119 & $+21,43$ \\
\hline & Longitudinale & 0,111 & $+13,30$ & 0,117 & $+19,40$ \\
\hline & Anisotrope & 0,114 & $+16,32$ & 0,121 & $+23,47$ \\
\hline
\end{tabular}

l'entrée du contact de 4 jusqu'à 24 microns. Les résultats obtenus montrent :

- une augmentation importante de la portance hydrodynamique avec le paramètre du couple de contrainte quelle que soit la valeur de $r$; cette augmentation est plus significative dans le cas des rugosités transversales et anisotropes ;

- une augmentation de la puissance dissipée avec le paramètre du couple de contrainte; cette augmentation est plus importante dans le cas des rugosités transversales ;

- une diminution du nombre de frottement avec le paramètre de couple de contrainte quelque soit la valeur de $r$.

Ces résultats montrent aussi que les couples de contrainte dus à la présence des additifs dans le lubrifiant n'ont pas d'effets appréciables sur le débit de fuite.

Les résultats illustrés dans la figure 10 montrent que les rugosités transversales permettent d'améliorer la 

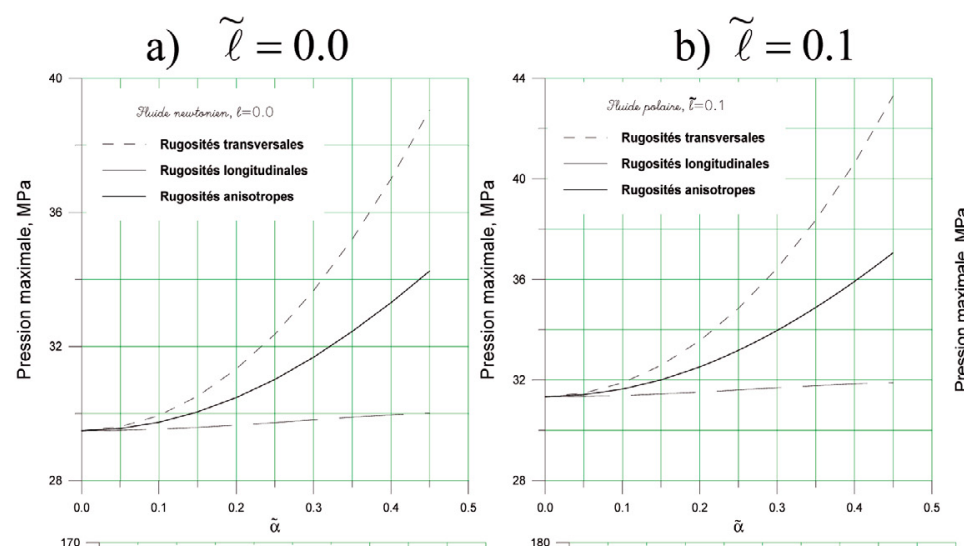
c) $\tilde{\ell}=0.3$
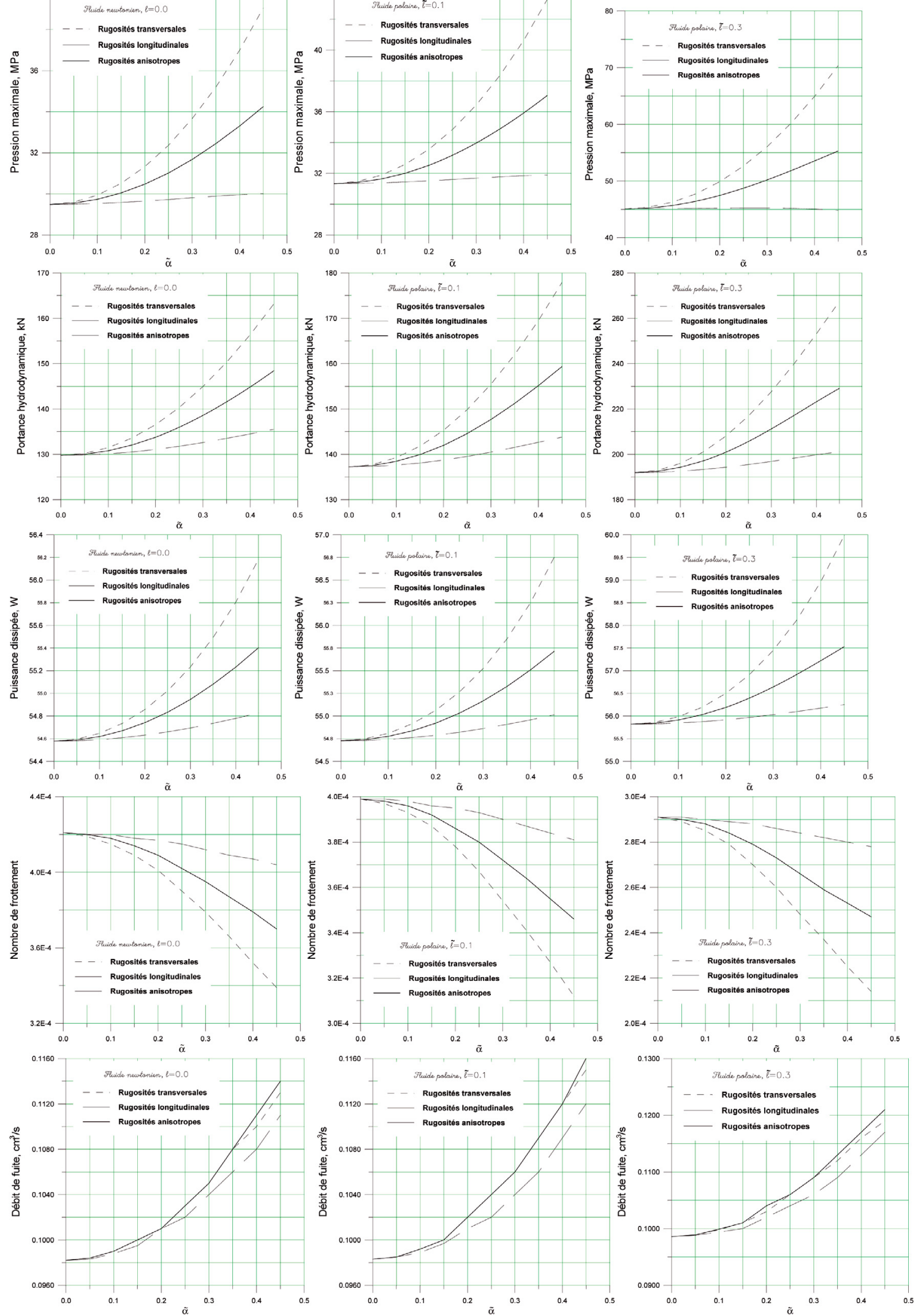

Fig. 8. Variations des performances hydrodynamiques du contact en fonction de l'amplitude relative des rugosités pour différentes valeurs du paramètre de couple de contrainte. 
(a)
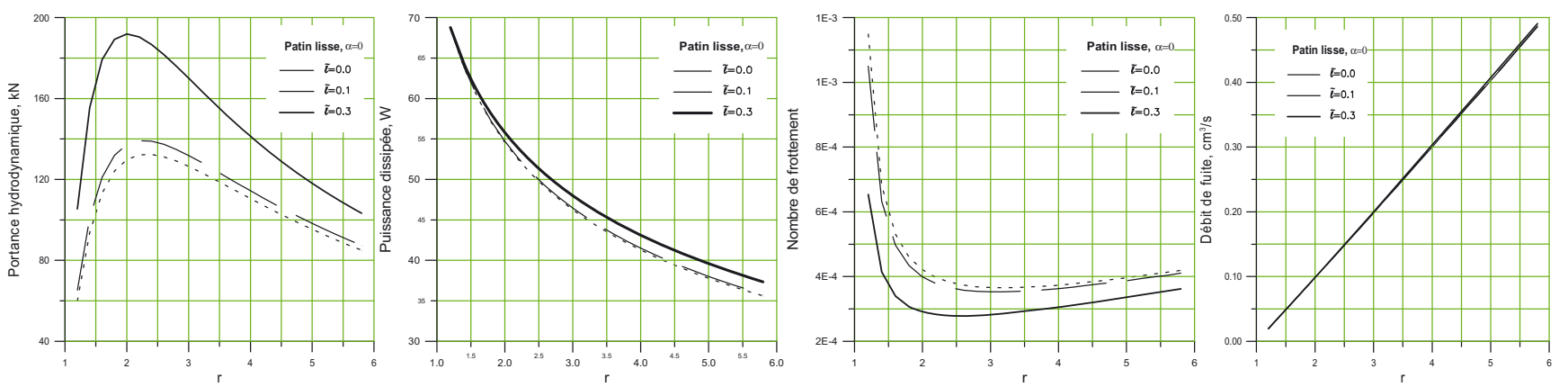

(b)
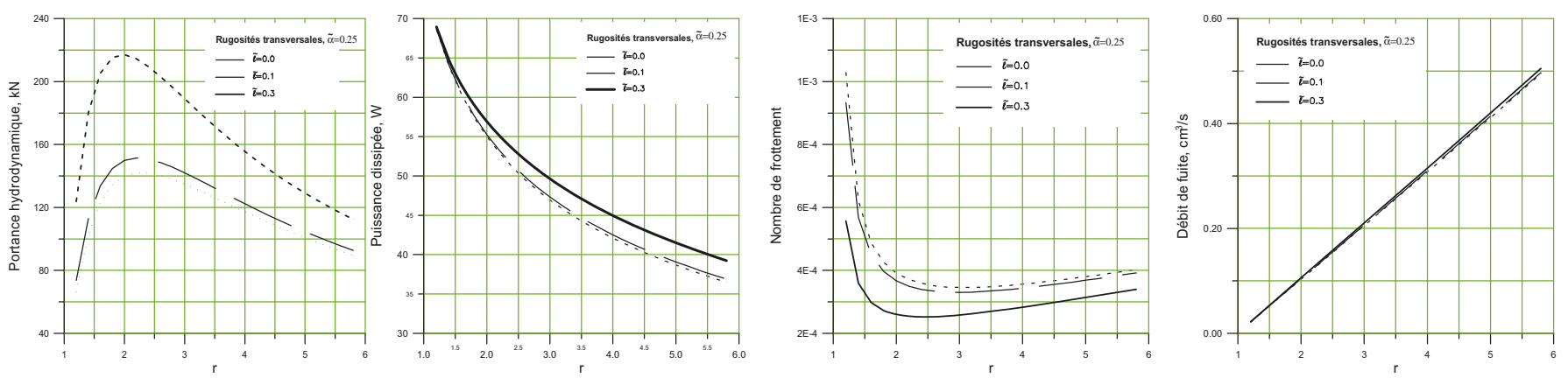

(c)
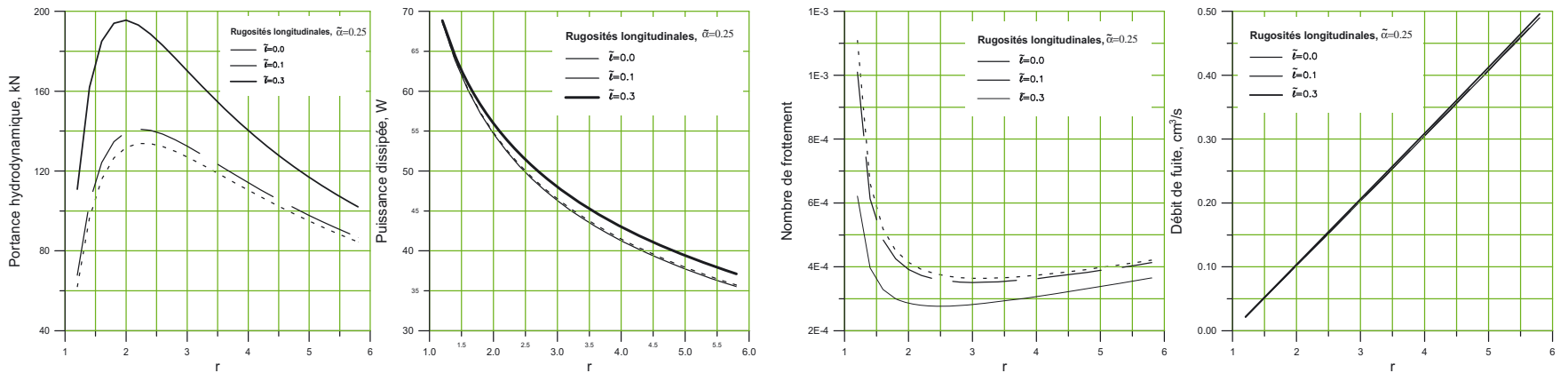

(d)
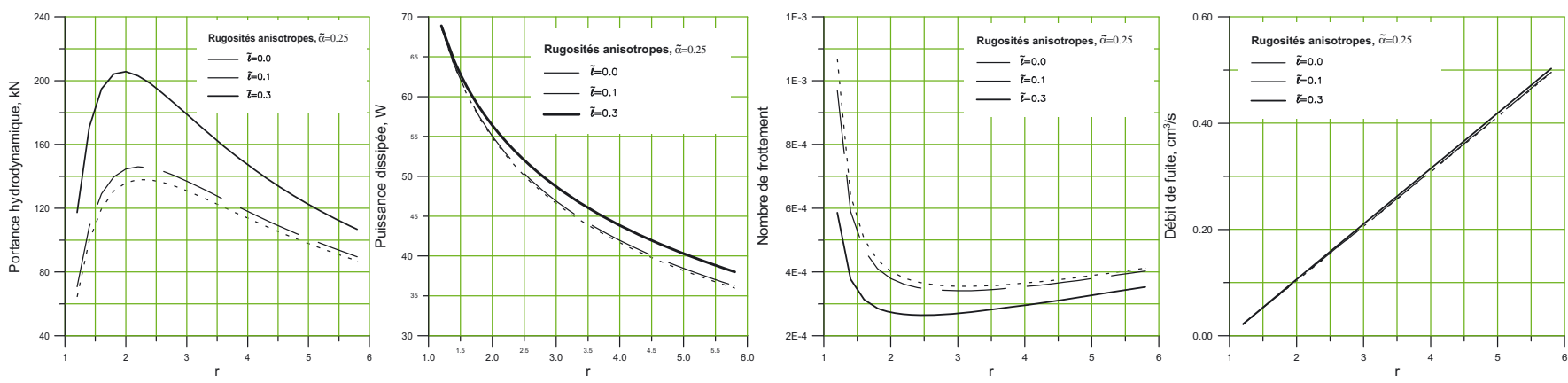

Fig. 9. Variations des performances hydrodynamiques du contact en fonction du rapport $r$ pour les différentes formes de rugosités. (a) Patin lisse. (b) Rugosités transversales. (c) Rugosités longitudinales. (d) Rugosités anisotropes. 
a) Portance hydrodynamique
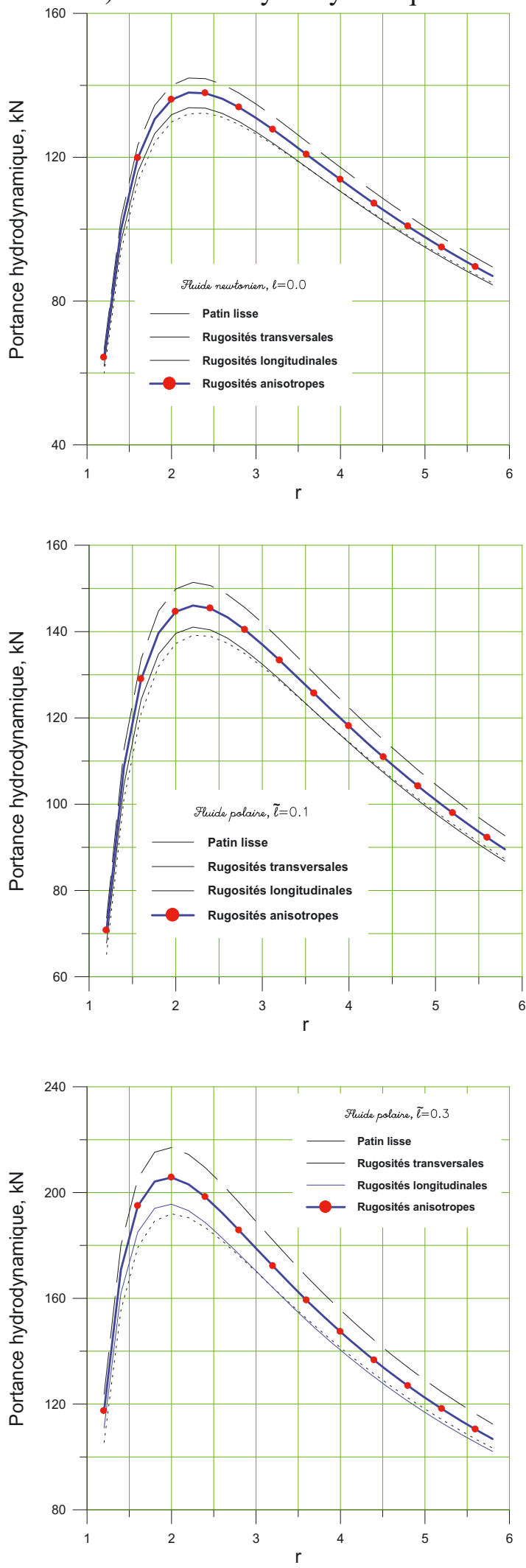

b) Nombre de frottement
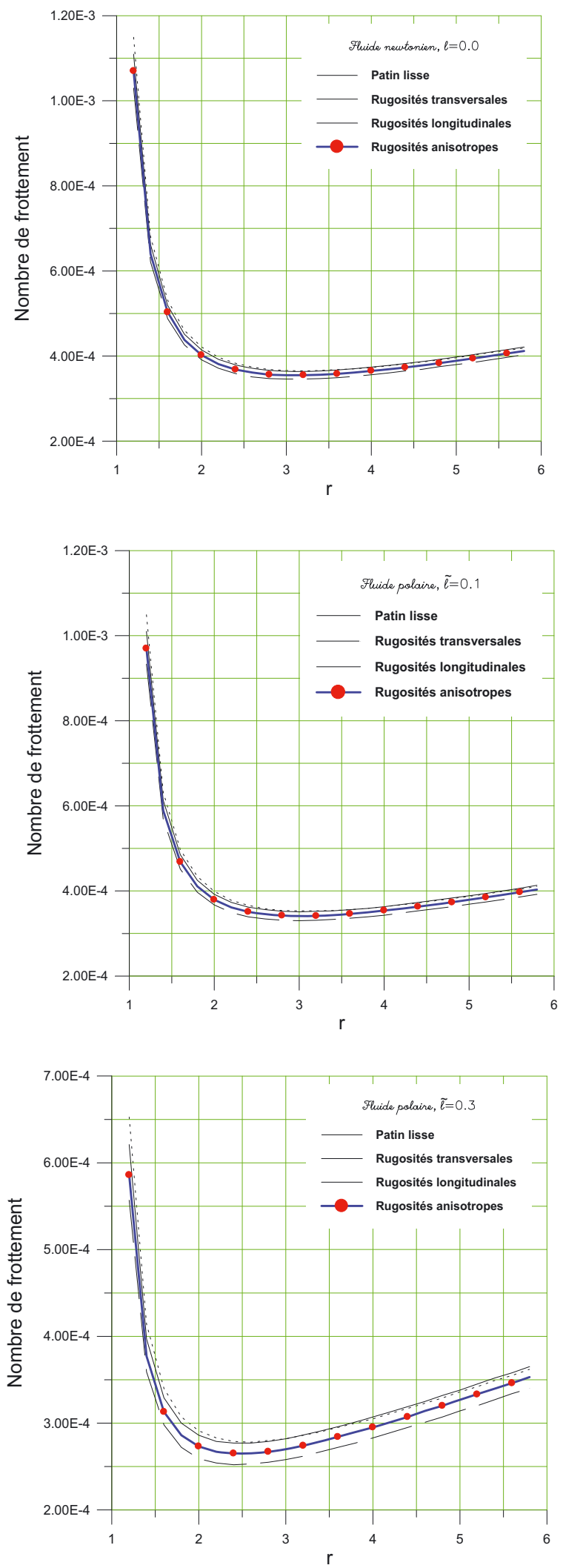

Fig. 10. Variations de la portance hydrodynamique du contact et du nombre de frottement en fonction du rapport $r$ pour les différentes formes de rugosités. 
capacité de charge du patin et de réduire le nombre de frottement.

Nous pouvons conclure que la présence des additifs de viscosité dans les huiles lubrifiantes permet d'améliorer la capacité de charge du patin et de réduire le frottement dans le contact surtout pour les additifs de longues chaînes moléculaires.

\section{Conclusions}

Dans la première partie de ce travail, nous avons étudié la convergence de la solution déterministe obtenue par la résolution numérique directe de l'équation de Reynolds vers la solution homogénéisée pour les trois formes de rugosités (transversales, longitudinales et anisotropes). La comparaison des solutions obtenues par les méthodes déterministe et d'homogénéisation a permis de conclure que la méthode d'homogénéisation est efficace du point de vue espace mémoire quelle que soit la forme de rugosité. En effet, pour des amplitudes relativement faibles et un nombre important de rugosités, la solution déterministe $p_{\varepsilon}$ obtenue par la résolution directe de l'équation de Reynolds newtonienne ou non newtonienne tend vers la solution homogénéisée $p_{0}$ pour les trois formes de rugosités considérées.

L'étude de l'influence des effets des couples de contrainte et des rugosités de surface effectuée en utilisant la méthode d'homogénéisation a conduit aux conclusions suivantes :

- La présence des rugosités entraîne une augmentation du pic de pression surtout dans le cas des rugosités transversales ce qui confirme bien les résultats obtenus par Dobrica et al. en utilisant les modèles déterministes et stochastiques [16].

- Pour les rugosités transversales et anisotropes, la pression maximale, la portance et le nombre de frottement sont sensiblement influencés par l'amplitude des rugosités aussi bien pour le cas newtonien que non newtonien, i.e. polaire. Cependant, ces paramètres ne sont pas affectés de façon significative par l'amplitude dans le cas des rugosités longitudinales.

- Pour une même valeur de l'amplitude, la pression maximale et la portance hydrodynamique calculées dans le cas des rugosités transversales sont supérieures à celles obtenues avec les rugosités anisotropes et longitudinales.

- La portance hydrodynamique augmente de façon importante avec le paramètre du couple de contrainte; cette augmentation est plus significative dans le cas des rugosités transversales et anisotropes.

- Une augmentation de la puissance dissipée avec le paramètre du couple de contrainte dans le cas lisse et rugueux.

- Le nombre de frottement diminue avec le paramètre du couple de contrainte.

- les couples de contrainte dus à la présence des additifs dans le lubrifiant n'affectent pas de façon significative le débit de fuite.

\section{Références}

[1] O. Reynolds, On the theory of lubrication and its application to M. Beauchamp Tower's experiments, Phil. Trans. Roy. Soc. London A 177 (1886) 157-234

[2] S.T. Tzeng, E. Saibel, Surface roughness effect on slider lubrication, ASLE Trans. 10 (1967) 334

[3] H. Christensen, Stochastics models for hydrodynamic lubrication of rough surfaces, Proc. IMechE 184 (Part 1) 1969-1970

[4] J. Prakash, K. Tiwari, Lubrication of a porous bearing with surface corrugations, ASME J. Lub. Tech. 104 (1982) 127-34

[5] H. Christensen, K. Tonder, The hydrodynamic lubrication of rough bearing surfaces of finite width, Trans. ASME J. Lub. Techn. 93 (1971) 324-330

[6] H.G. Elrod, Thin-film lubrication theory for Newtonian fluids possessing striated roughness or grooving, ASME J. Lub. Tech. 95 (1973) 484-489

[7] N. Patir, H.S. Cheng, Effect of surface roughness orientation on central film thickness in EHD contact, Proc. 5th Leeds-Lyon Symp. Trib., 1978, pp. 15-21

[8] S. Boedo, J.F. Booker, Surface roughness and structural inertia in a mode-based mass conserving elastohydrodynamic lubrication model, Trans. ASME J. Trib. 119 (1997) 449-455

[9] P. Wang, T.G. Keith, Combined surface roughness of dynamically loaded journal bearings, Trib. Trans. 45 (2002) $1-10$

[10] J.R. Elrod, A cavitation algorithm, Trans. ASME J. Lub. Tech. 103 (1981) 350-354

[11] D. Vijayaraghavan, J.R. Keith, Development and evaluation of a cavitation algorithm, Trib. Trans. 32 (1989) $225-233$

[12] N.B. Naduvinamani, A. Siddangouda, Effect of surface roughness on the hydrodynamic lubrication of porous step-slider bearings with couple stress fluids, Trib. Int. 40 (2007) 780-793

[13] C.C. Kweh, M.J. Patching, H.P. Evans, R.W. Snidle, Simulation of elastohydrodynamic contacts between rough surfaces, Trans. ASME J. Trib. 114 (1992) 412-419

[14] J.A. Greenwood, Morales, G.E. Espejel, The behavior of transverse roughness in elastohydrodynamic lubrication contact, Proc. of the IMechE J. Trib. Eng. Part J 208 (1994) 121-132.

[15] D. Zhu, X. Ai, Point contact elastohydrodynamic lubrication based on optically measured three-dimensional rough surfaces, Trans. ASME J. Trib. 119 (1997) 375-384

[16] M.B. Dobrica, M. Fillon, P. Maspeyrot, Mixed elastohydrodynamic lubrication in partial journal bearings comparison between deterministic and stochastic models, ASME J. Trib. 128 (2006) 778-788

[17] M.B. Dobrica, M. Fillon, P. Maspeyrot, Influence of mixed lubrication and rough elastic-plastic contact on the performance of small fluid film bearings, STLE Trib. Trans. 51 (2008) 699-717

[18] V.A. Marchenko, E.Ya. Khruslov, Homogenization of partial differential equations, Birkhäuser, Boston, 2006

[19] O. Hiroshi, F. Yasuyoshi, K. Noriyoshi, Homogenization method for heterogeneous material based on boundary element method, Comput. Struct. 79 (2001) 1987-2007 
[20] K. Marcin, Sensitivity and randomness in homogenization of periodic fiber-reinforced composites via the response function method, Int. J. Solids Struct. 46 (2009) 923-937

[21] G. Bayada, J.B. Faure, A double scale analysis approach of the Reynolds roughness comments and application to the journal bearing, J. Trib. 111 (1989) 323-330

[22] M. Jai, Homogenization and two-scale convergence of the compressible Reynolds lubrication equation modelling the flying characteristics of a rough magnetic head over a rough rigid-disk surface, ASME J. Trib. 124 (2002) 327-335

[23] G. Bayada, S. Ciuperca, M. Jai, Homogenization of variational equations and inequalities with small oscillating parameters: Application to the study of thin film unstationary lubrication flow, C. R. Acad. Sci. Paris, t. 328, Série II b (2000) 819-824

[24] M. Jai, B. Bou-Said, A comparison of homogenization and averaging techniques for the treatment of roughness in slip-flow-modified Reynolds equation, Trans. ASME J. Trib. 124 (2002) 327-335

[25] G.C. Buscaglia, M. Jai, Homogenization of the generalized Reynolds equation for ultra-thin gas films and its resolution by FEM, J. Trib. 126 (2004) 547-552

[26] M. Kane, B. Bou-Said, Comparison of homogenization and direct techniques for the treatment of roughness in incompressible lubrication, J. Trib. 126 (2004) 733-737

[27] M. Kane, B. Bou-Said, A study of roughness and nonnewtonian effects in lubricated contacts, ASME J. Trib. 127 (2005) 575-581

[28] A. Almqvist, D. Lukkassen, A. Meidell, P. Wall, New concepts of homogenization applied in rough surface hydrodynamic lubrication, Int. J. Eng. Sci. 45 (2007) 139-154

[29] A. Almqvist, E.K. Essel, L.E. Persson, P. Wall, Homogenization of the unstationary incompressible Reynolds equation, Trib. Int. 40 (2007) 1344-1350

[30] V.K. Stokes, Couple stresses in fluids, Phys. Fluids 9 (1966) 1709-1715

[31] M. Lahmar, B. Bou-Saïd, Couple-stresses effects on the dynamic behavior of connecting-rod bearings in both gosoline and diesel engines, J. Trib. Trans. STLE 51 (2008) $44-56$

[32] A. Kabouya, M. Lahmar, B. Bou-Saïd, Étude des paliers lisses mésalignés lubrifiés par des fluides à couple de contrainte, Mécanique \& Industries 8 (2007) 577-595

[33] H. Boucherit, M. Lahmar, B. Bou-Saïd, Misalignment effect on steady-state and dynamic behaviour of compliant journal bearings lubricated with couple stress fluids, J. Lubr. Sci. 20 (2008) 241-268

[34] R.S. Gupta, L.G. Sharma, Analysis of couple stress lubricant in hydrostatic thrust bearings, Wear 48 (1988) 257-269

[35] J.R. Lin, Static and dynamic characteristics of externally pressurized circular step thrust bearings lubricated with couple stress fluids, Trib. Int. 32 (1999) 207-216

[36] P. Sinha, C. Singh, Couple stresses in the lubrication of rolling contact bearings considering cavitation, Wear 67 (1981) 85-91

[37] N.M. Bujurke, N.G. Naduvinami, The lubrication of the lightly cylinders in combined rolling, sliding and normal motion with couple stress fluid, Int. Mech. Sci. 32 (1990) 969-979

[38] J.R. Lin, Squeeze film characteristics of long partial journal bearings lubricated with couple stress fluids, Trib. Int. 30 (1997) 53-58

[39] J.R. Lin, Squeeze film characteristics of finite journal bearings : couple stress fluid model, Trib. Int. 4 (1998) 201-207

[40] J.R. Lin, C.B. Yang, R.F. Lu, Effects of couple stresses in the cyclic squeeze films of finite partial journal bearings, Trib. Int. 34 (2001) 119-125

[41] N.B. Naduvinamani, P.S. Hiremath, G. Gurubasavaraj, Squeeze film lubrication of a short porous journal bearing with couple stress fluids, Trib. Int. 34 (2001) 739-747

[42] U.M. Mokhiamar, W.A. Crosby, H.A. El-Gamal, A study of a journal bearing lubricated by fluids with couple stress considering the elasticity of the liner, Wear 224 (1999) 194-201 